\title{
4D-CT Parathyroid for the General Radiologist: A Pictorial Essay of Illustrative Cases
}

Julia Gosis MD, Ali Sepahdari MD, Stellios Karnezis MD, Avetis Azizyan MD, Noriko Salamon MD PhD, Arvind Vijayasarathi MD MBA MPH

\author{
Division of Neuroradiology \\ Department of Radiological Sciences \\ David Geffen School of Medicine at UCLA
}




\section{Disclosures}

\section{The authors have no relevant financial or nonfinancial relationships to disclose.}




\section{Educational Objectives}

- Describe the purpose of 4D-CT for evaluation of primary hyperparathyroidism and pre-surgical planning

- Review 4D-CT imaging protocol and illustrate the typical appearance of parathyroid adenomas

- Review the embryology of the parathyroid glands, and use it to facilitate a systematic search for embryologic and ectopic adenomas

- Illustrate a variety of cases of parathyroid adenomas in embryologic and ectopic locations and cases of multi-glandular disease

- Illustrate important mimics of parathyroid disease and important "incidental" findings in the neck and upper chest 


\section{Hyperparathyroidism}

- Increased parathyroid hormone causes increased osteoclastic activity

- Subtypes

- Primary

- Parathyroid adenoma

- Parathyroid hyperplasia

- Parathyroid carcinoma

- Secondary

- Caused by chronic hypocalcemia

- Most often due to renal osteodystrophy, vitamin D deficiency or malnutrition

- Leads to increased parathyroid hormone levels

- Tertiary

- Autonomous adenoma caused by chronic overstimulation of hyperplastic glands, usually in the setting of renal insufficiency 


\section{Hyperparathyroidism}

- 3 to 4 times more common in women than men

- 50-70 most common age range

- Majority of cases of primary hyperparathyroidism are caused by adenomas, ( $90 \%)$

- Less commonly there are multiple adenomas ( $\sim 5 \%)$, multigland hyperplasia $(\sim 5 \%)$, or parathyroid carcinoma $(<1 \%)$

- Approximately $20 \%$ of adenomas are in ectopic locations (numbers vary in literature)

- Surgical excision of adenoma is treatment of choice in primary hyperparathyroidism 


\section{Hyperparathyroidism}

- Historically, multigland exploration/excision was performed without pre-operative imaging

- In the early 2000s, minimally invasive resection emerged, and preop planning importance rose

- Single adenoma resected through small incision; remaining glands left unexplored if PTH level drops

- Radiologist role is to:

- Describe/localize high suspicion nodules

- Identify ectopic adenomas

- Recognize multigland hyperplasia or multiple adenomas

- Highlight any relevant anatomical considerations 


\section{Imaging Technique}

- Ultrasound

- Inexpensive, fast, can be done by surgeons in office to localize typical adenomas greater than $1 \mathrm{~cm}$.

- Dual phase Tc-99m Sestamibi scan

- Early images 10-20 min post injection (activity in both thyroid \& parathyroid)

- Delayed (90 mins), activity in thyroid washes out, persists in adenoma

- Can do SPECT/CT for better localization

- 4D CT parathyroid

- Improved sensitivity of localization, particularly in ectopic/mediastinal locations

- Helpful for pre-operative planning 


\section{CT parathyroid technique}

- "4D" - axial, sagittal, coronal, and dynamic enhancement characteristics

- Precontrast

- Arterial phase (25-30 seconds after injection of contrast commences)

- Venous phase (60-80 seconds after injection of contrast commences)

- At UCLA, our arterial phase extends from skull base to carina to help identify ectopic locations

- All three phases and planes should be evaluated; however the arterial phase is usually the most helpful 


\section{Anatomy}

- Embryology is relevant to parathyroid adenomas

- Inferior glands (paired) arise from the $3^{\text {rd }}$ branchial pouch

- Superior glands (paired) arise from the $4^{\text {th }}$ branchial pouch

- These pouches are approximately at level of carotid bifurcation

- Parathyroid glands descend during development

- Inferior glands migrate with the thymus, and can be found from carotid bifurcation to carina, including within the thymus or thyroid gland

- Superior glands migrate with thyroid gland (less variable location) 


\section{Anatomy}

- Embryologic superior glands are located posterior to the upper third of the thyroid gland, often paraesophageal or within the tracheoesophageal groove

- Embryologic superior glands can fall caudally (descended) and can be found as low as within the mediastinum (typically posterior in location)

- Embryologic inferior glands are usually closely associated with the inferior thyroid (immediately inferior, posterior, lateral or anterior to the tip of the gland).

- Embryologic inferior glands are located more anteriorly (paratracheal, anterior mediastinum, closely associated with strap musculature) 


\section{Important to mention: Aberrant SCA}
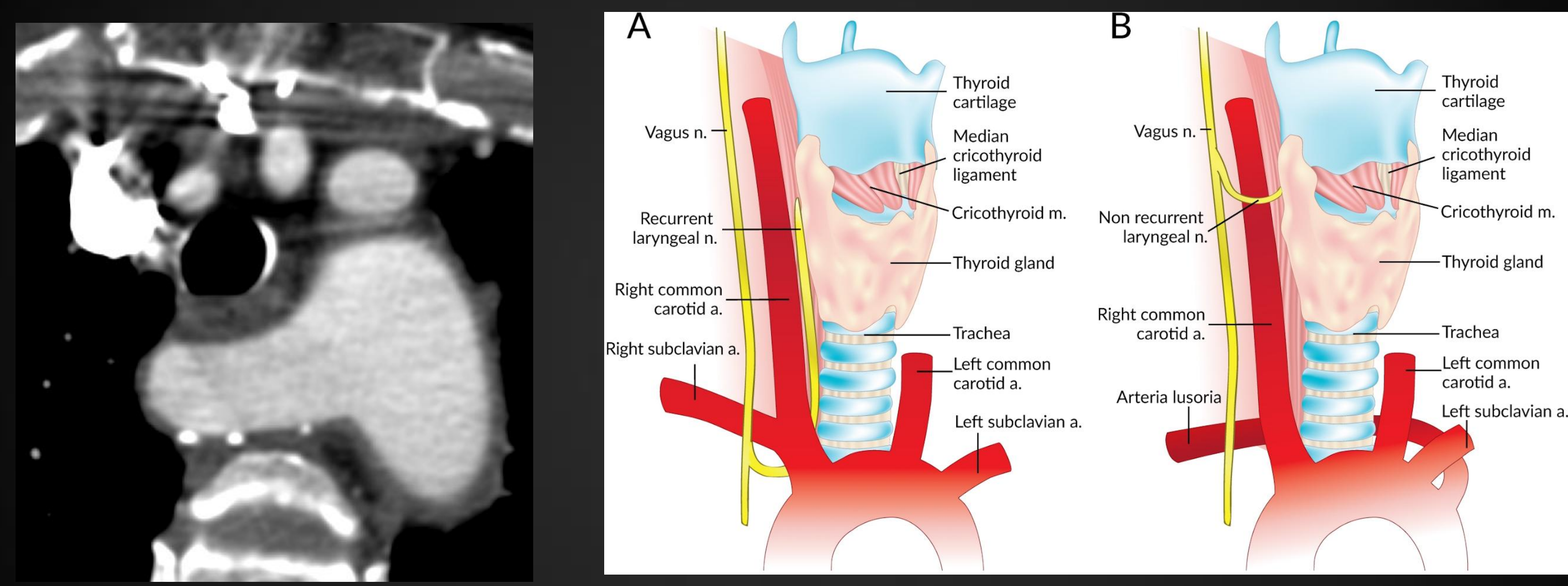

Aberrant SCA associated w/ nonrecurrent laryngeal nerve (not in TE groove)! 


\section{Enhancement Characteristics}

- Typical parathyroid adenomas are:

- Hypoattenuating to thyroid on precontrast

- Arterially hyperenhancing

- Rapid washout on venous phase

- Precontrast sequence is very important to differentiate thyroid tissue from parathyroid

- Start with arterial phase to search for possible adenoma, and then confirm on precontrast and delayed phase

- DDx of possible adenomas include thyroid (use precontrast to distinguish), lymph nodes (typically progressively enhance on venous phase, do not wash out) 


\section{Enhancement Characteristics}

- Caveats:

- In approximately one third of cases an adenoma will be isodense to thyroid on arterial and venous phases

- Patients with hypothyroidism demonstrate a lower intrinsic attenuation of the thyroid gland limiting the usefulness of the pre-contrast scan (these patients do not organify iodine as those with normally functioning thyroid glands)

- Cystic degeneration of an adenoma, streak artifact, suboptimal bolus timing, thyroiditis can alter enhancement characteristics 


\section{CASES}


Case 1 


\section{Case 1}

- Right sided embryologic inferior parathyroid adenoma

- Along inferior margin of right lobe of thyroid

- Paratracheal, closely associated with the overlying strap musculature

- Hypodense to thyroid on precontrast (A, D), arterially enhancing $(B, E)$, and demonstrates washout on venous phase (C, F) 
Case 2
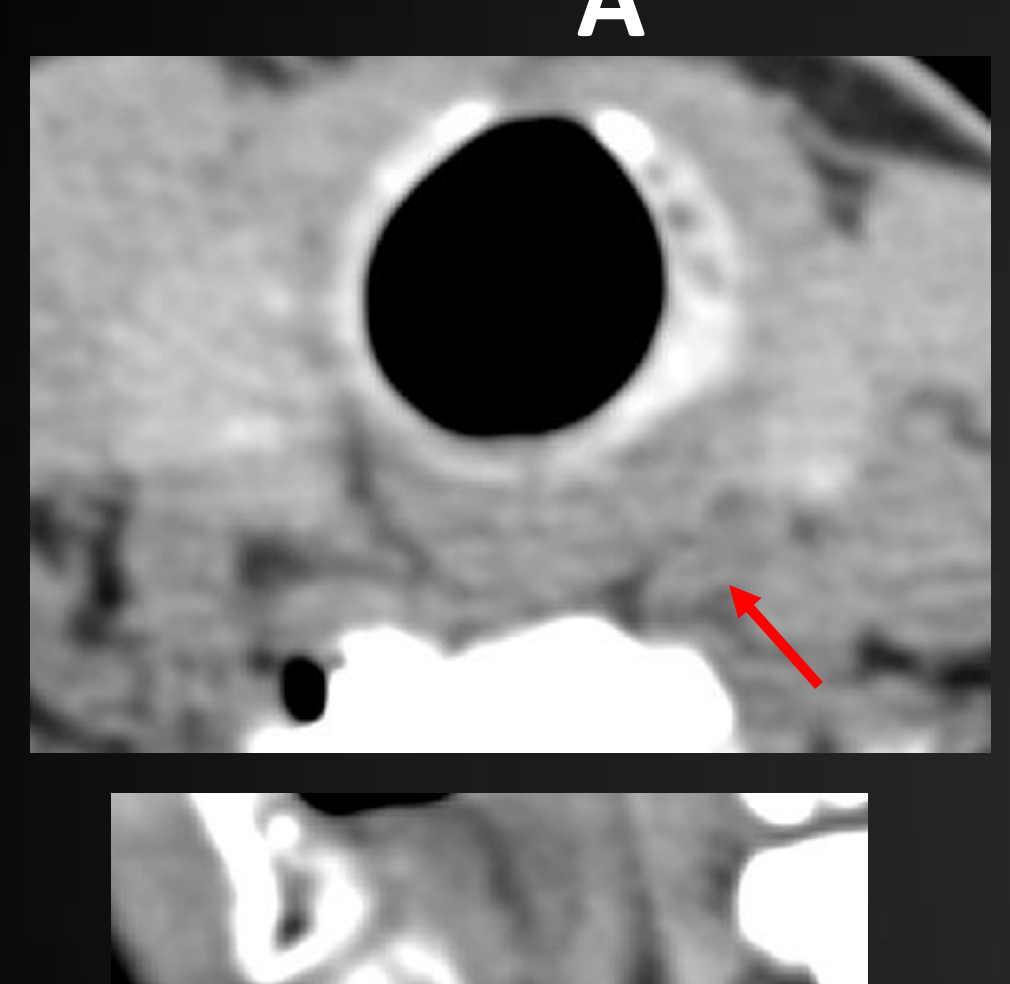

D
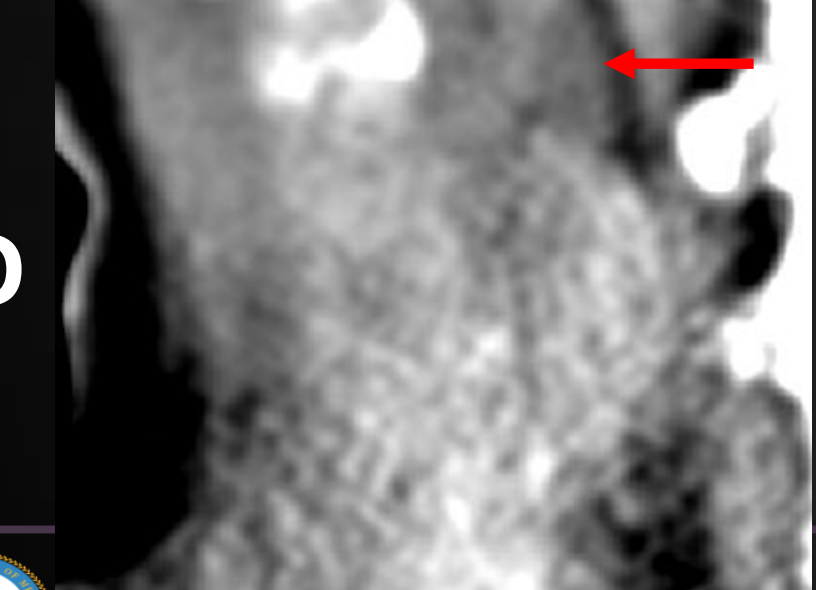

B

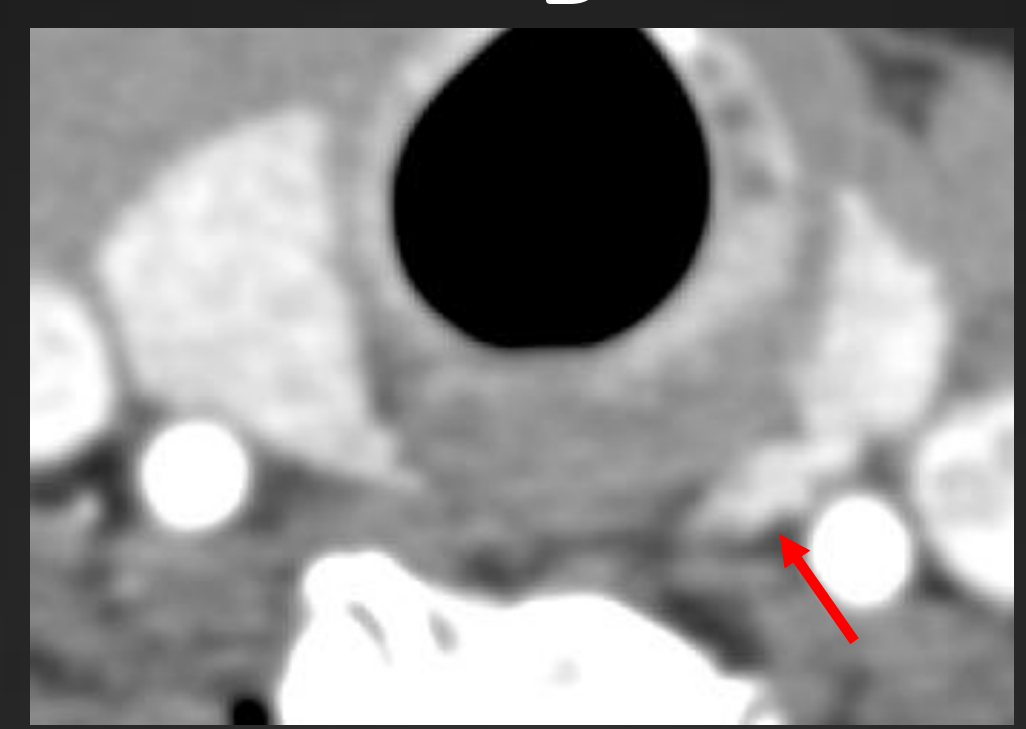

E

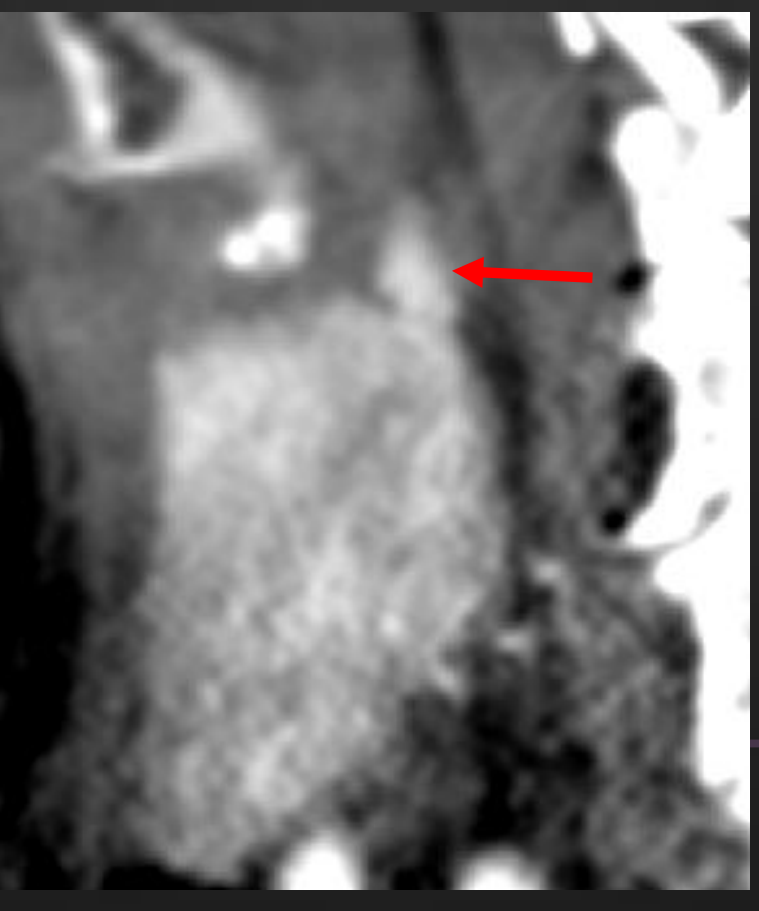

C

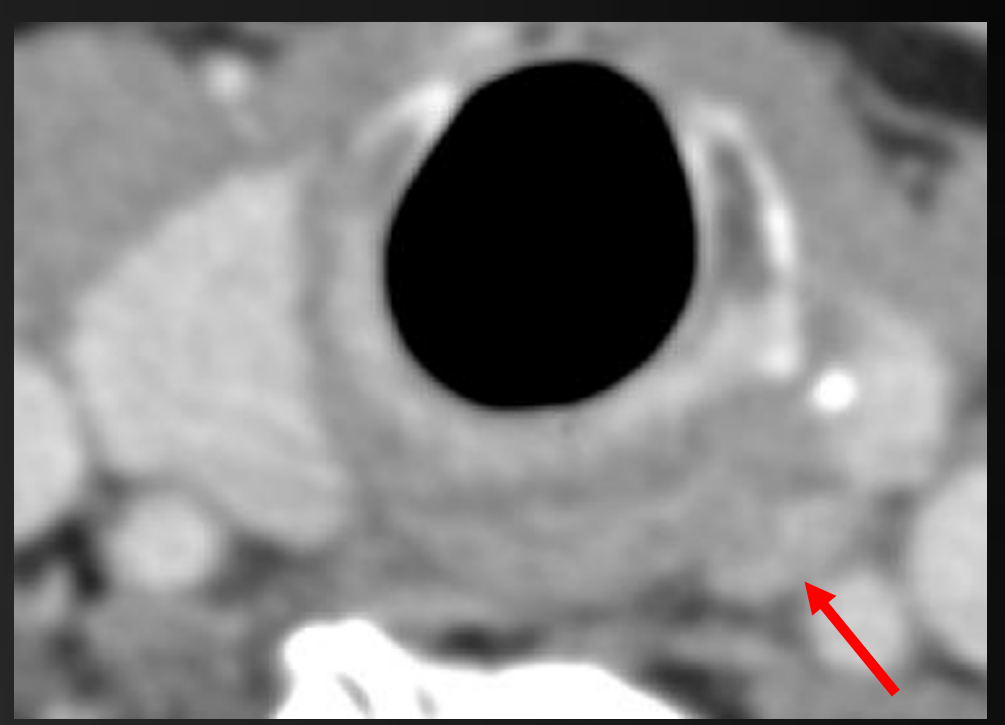

F
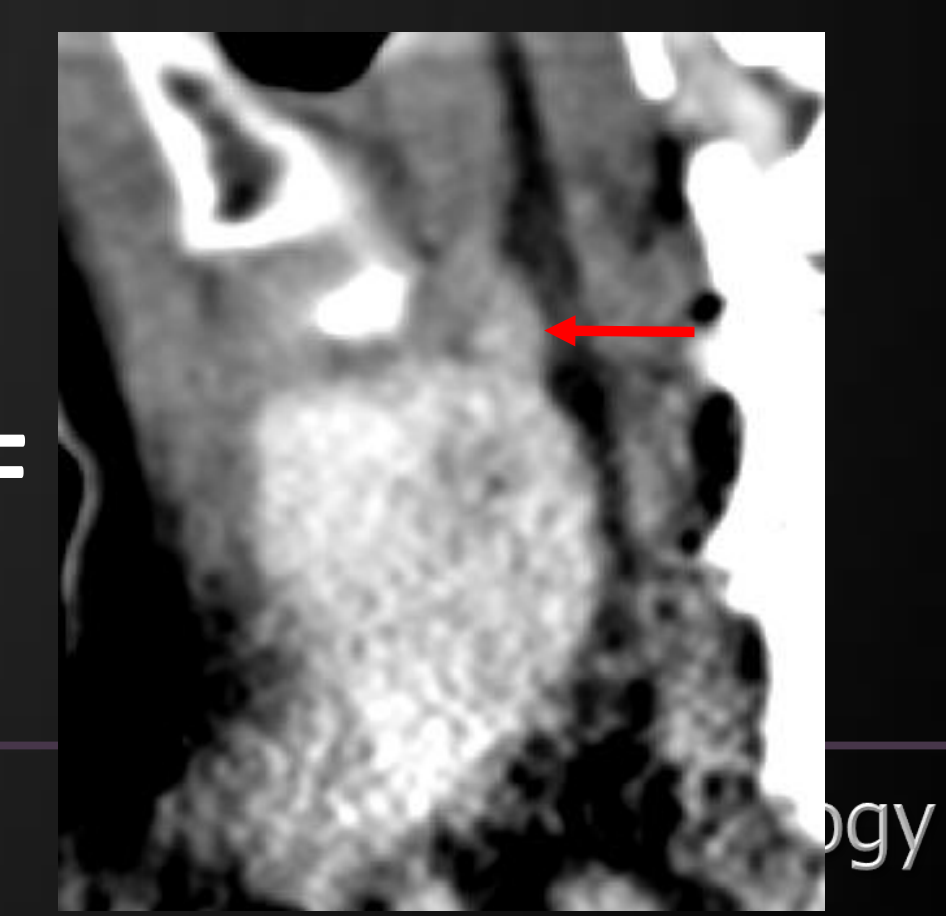


\section{Case 2}

- Embryologic superior left parathyroid adenoma

- Paraesophageal, posterior to the superior aspect of the thyroid lobe

- Hypodense to thyroid on precontrast (A, D), arterially enhancing $(B, E)$, demonstrates washout on venous phase $(C, F)$ 


\section{Case 3}

A

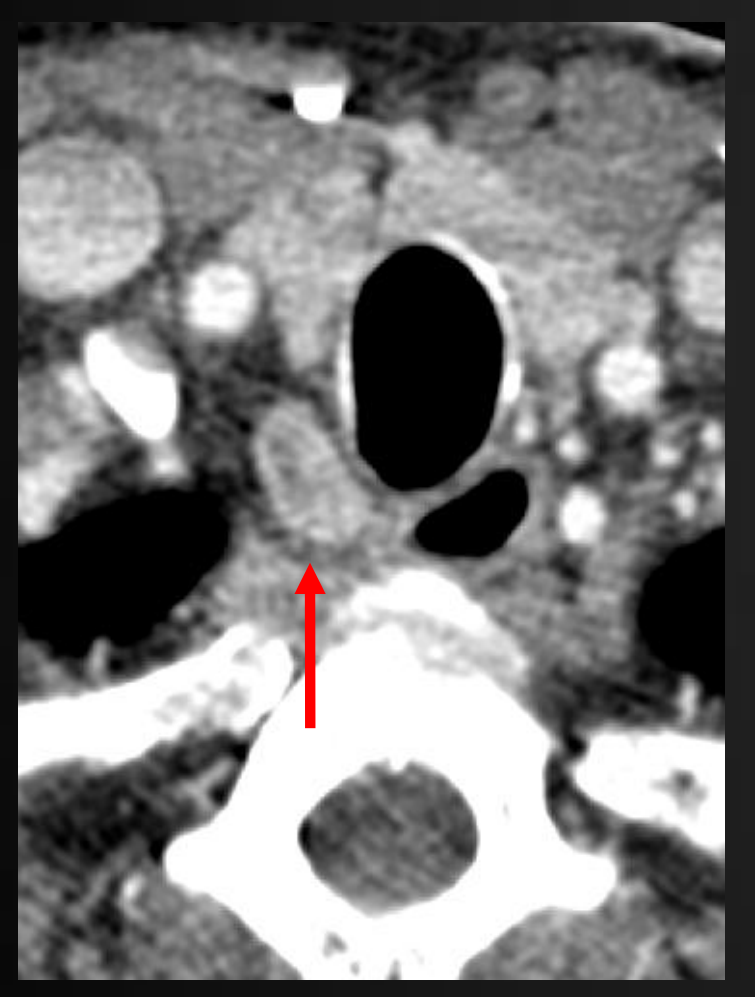

B

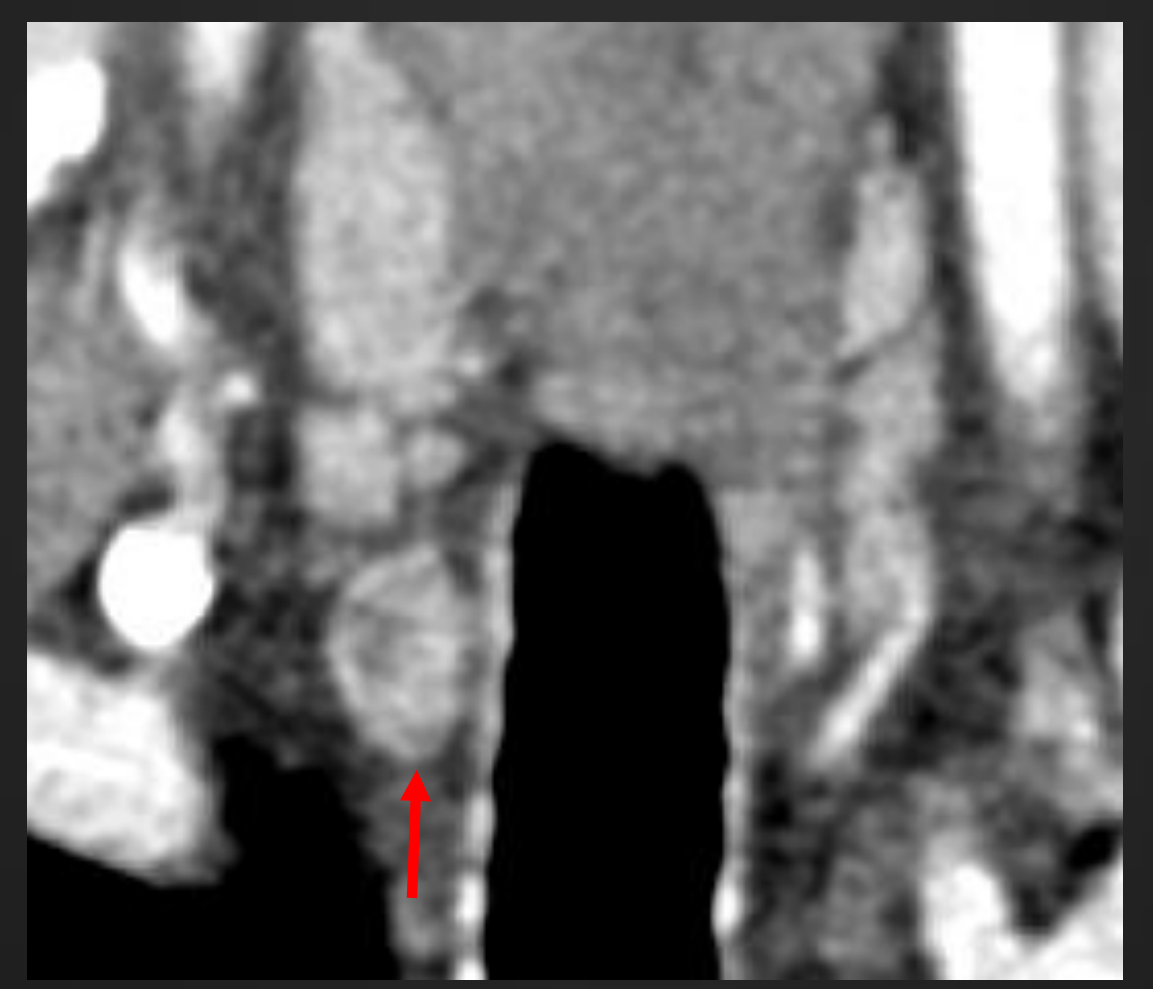

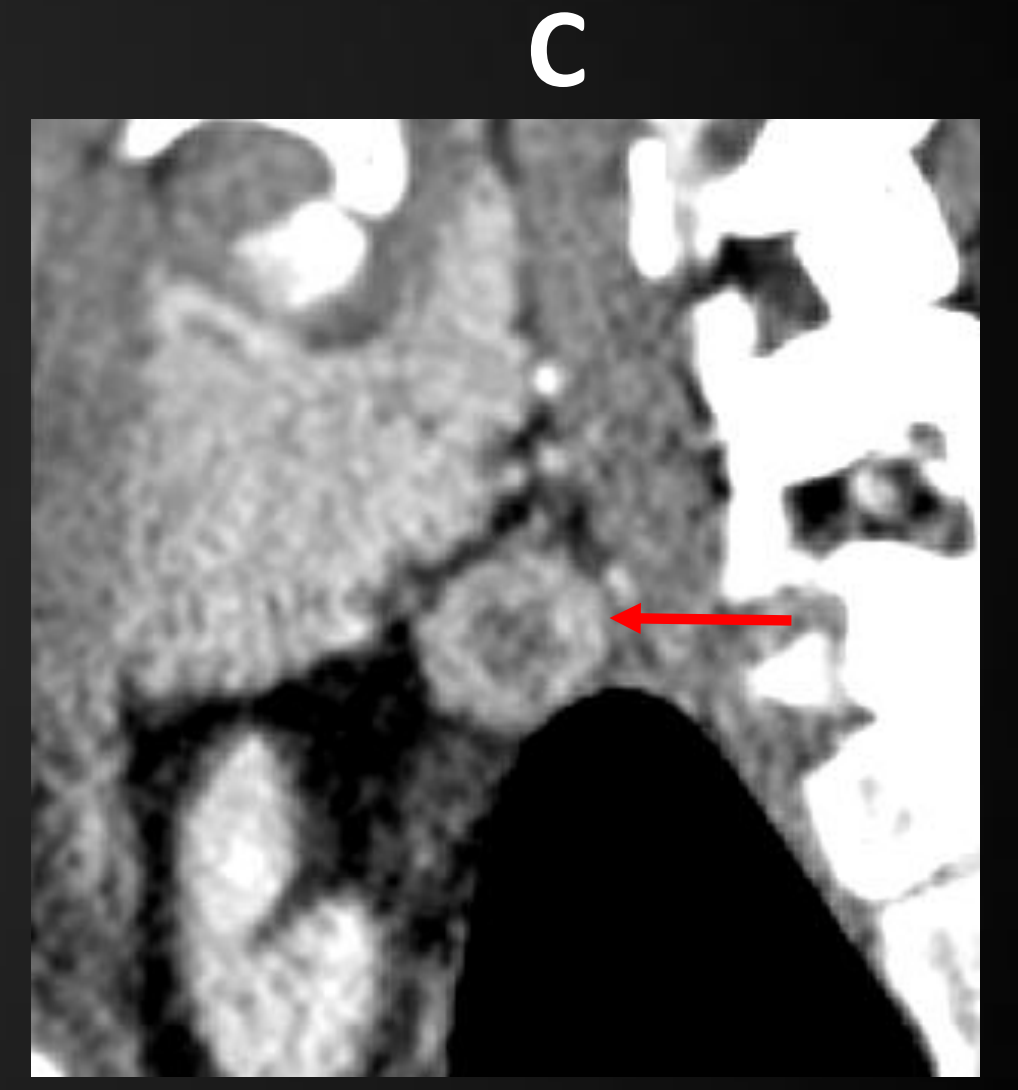

UCLA Radiology
UCLA Health 


\section{Case 3}

- Right sided descended embryologic superior gland adenoma

- Parathyroid adenoma in right tracheoesophageal groove, along inferior aspect of the right thyroid lobe

- Arterial enhancement on axial (A), coronal (B), and sagittal (C) images with internal cystic change 


\section{Case 4}
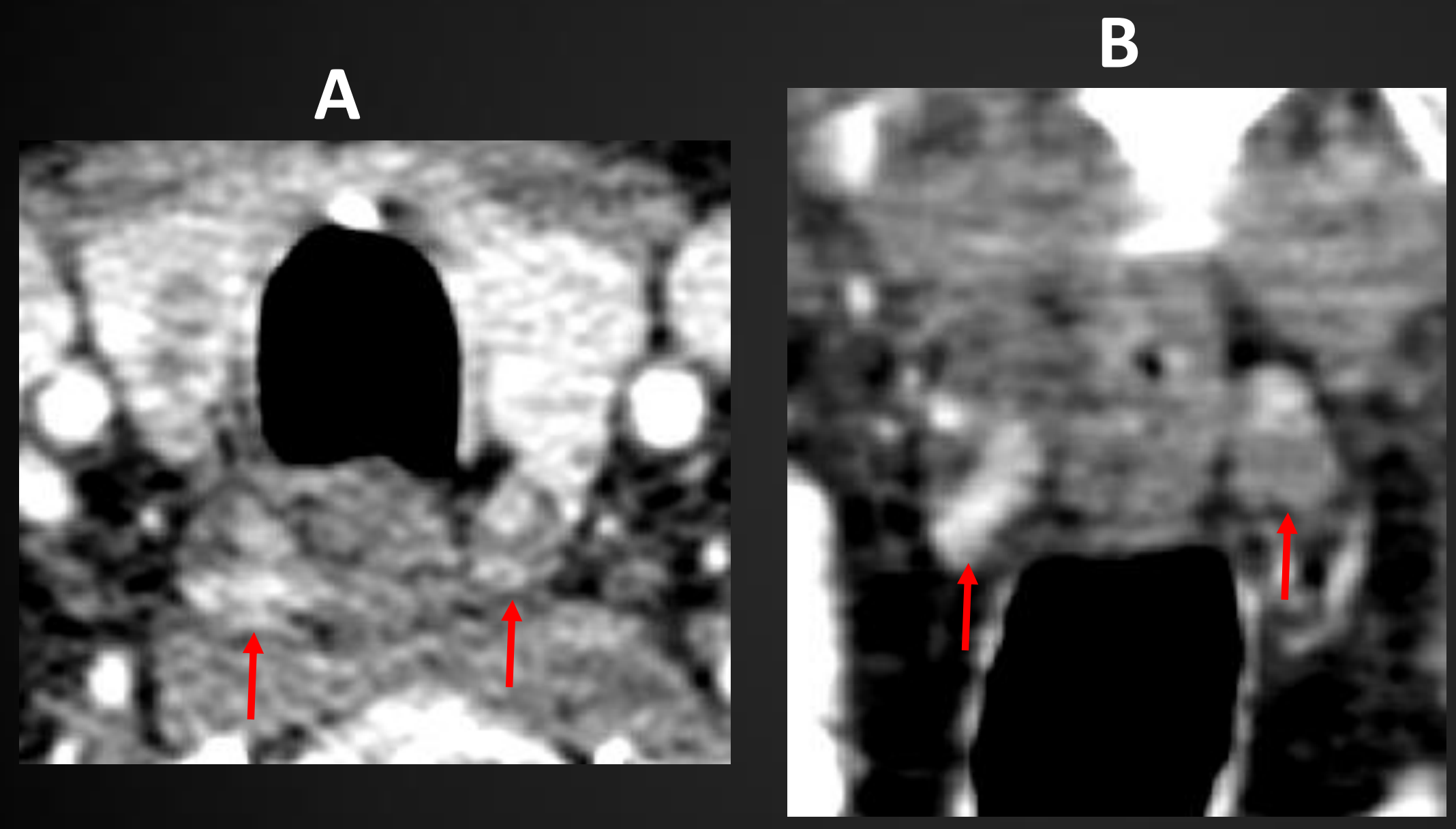

c
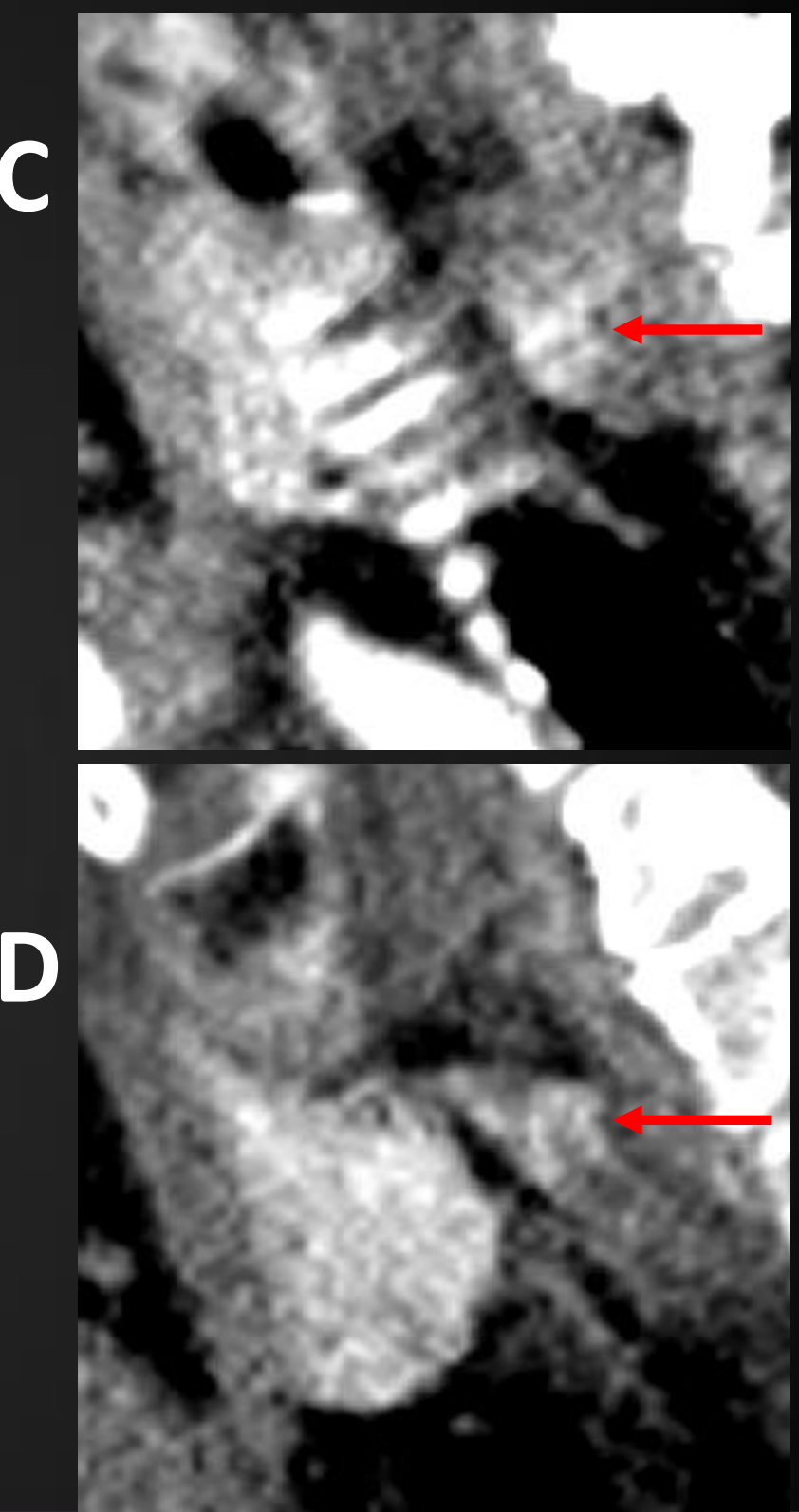


\section{Case 4}

- Bilateral embryologic superior gland parathyroid adenomas

- Arterial enhancement on axial (A), coronal (B), and off-midline sagittal (C, D) images 


\section{Case 5}

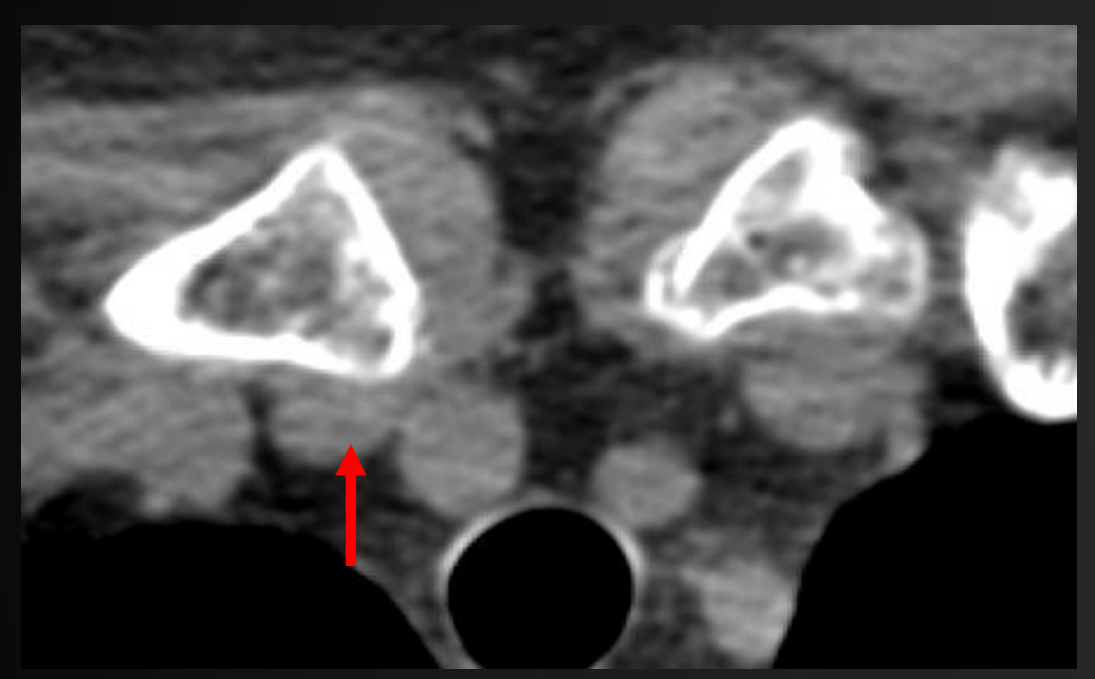

A

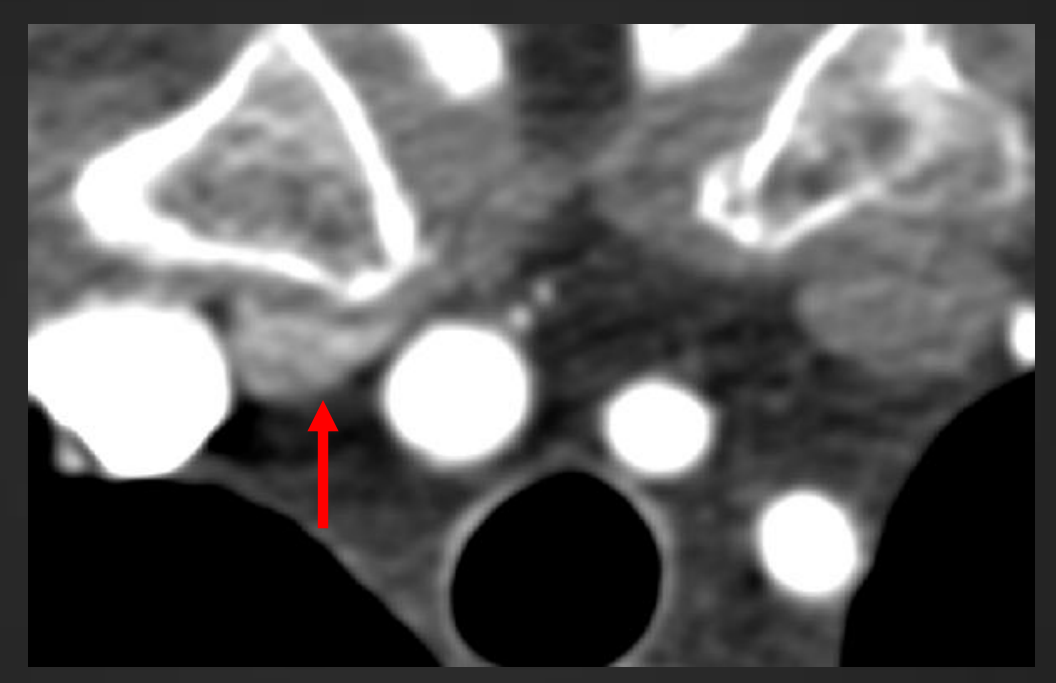

B

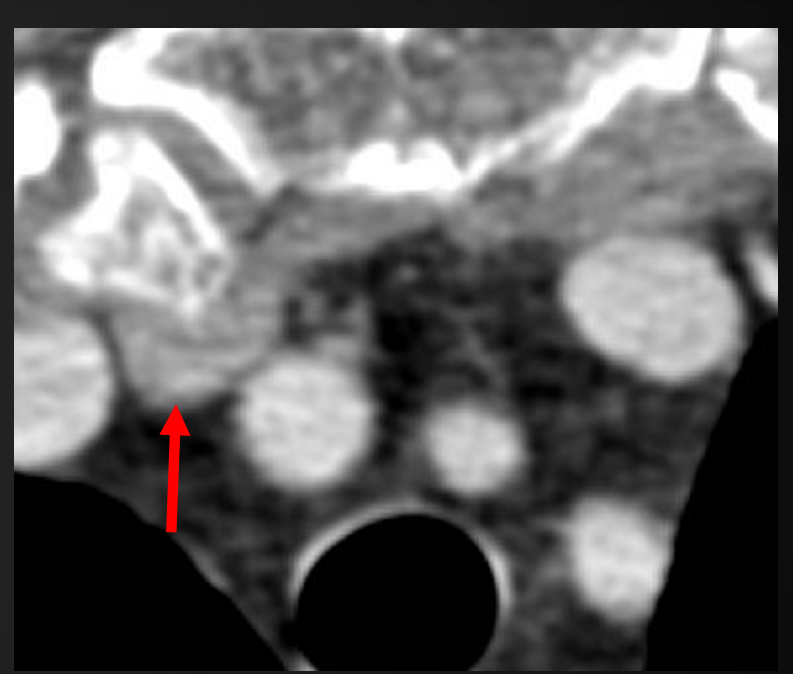

C 
Case 5

D

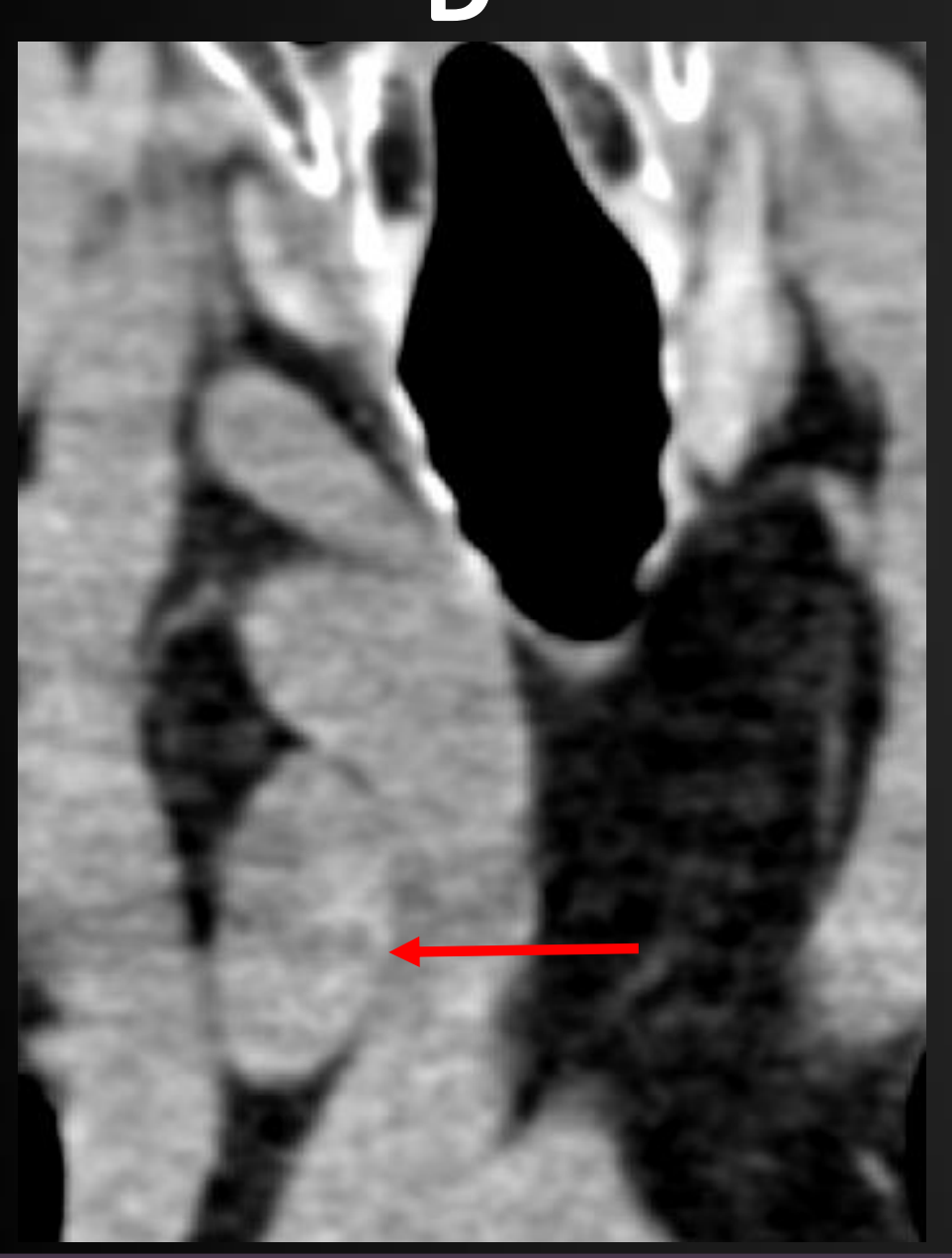

E

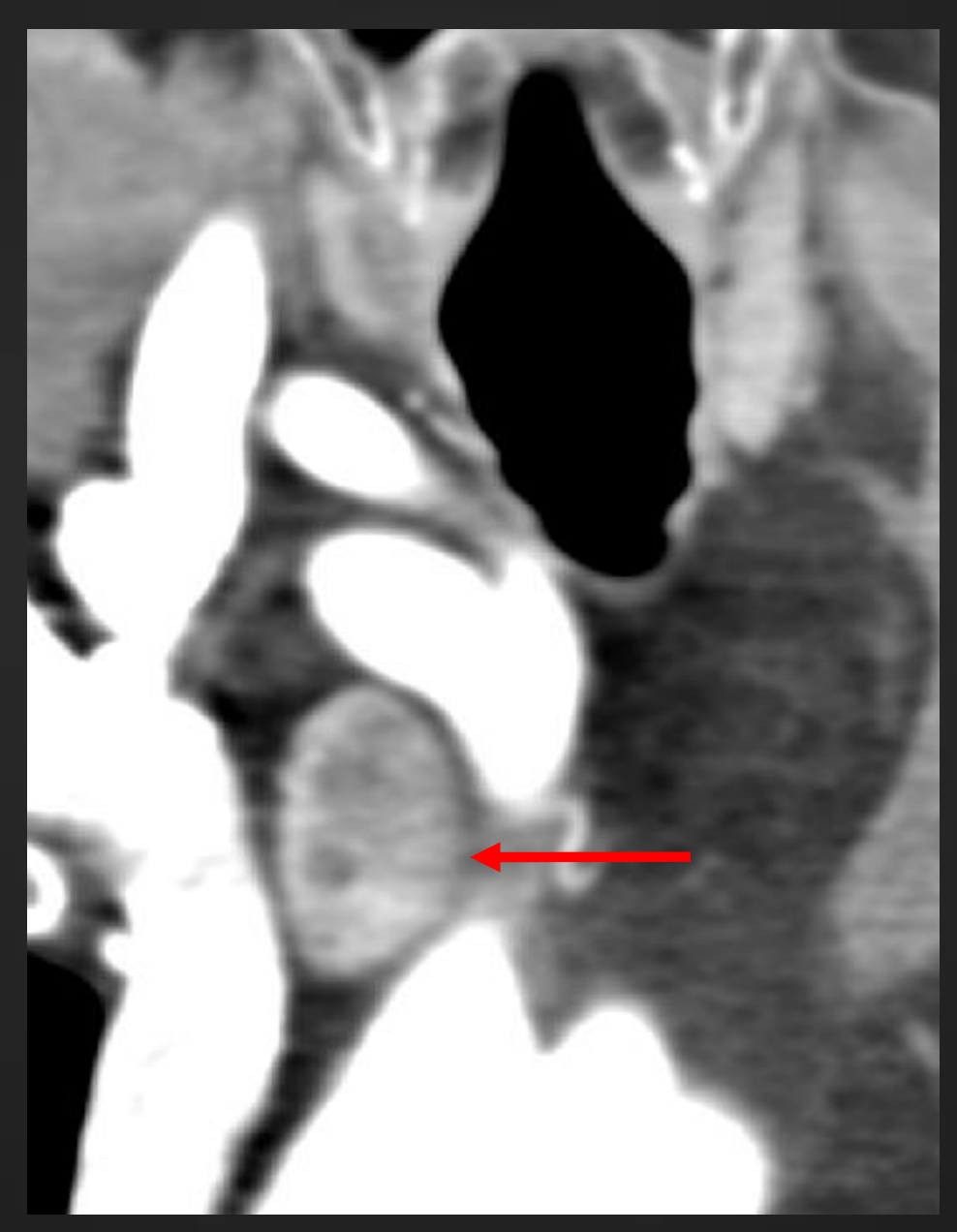

F

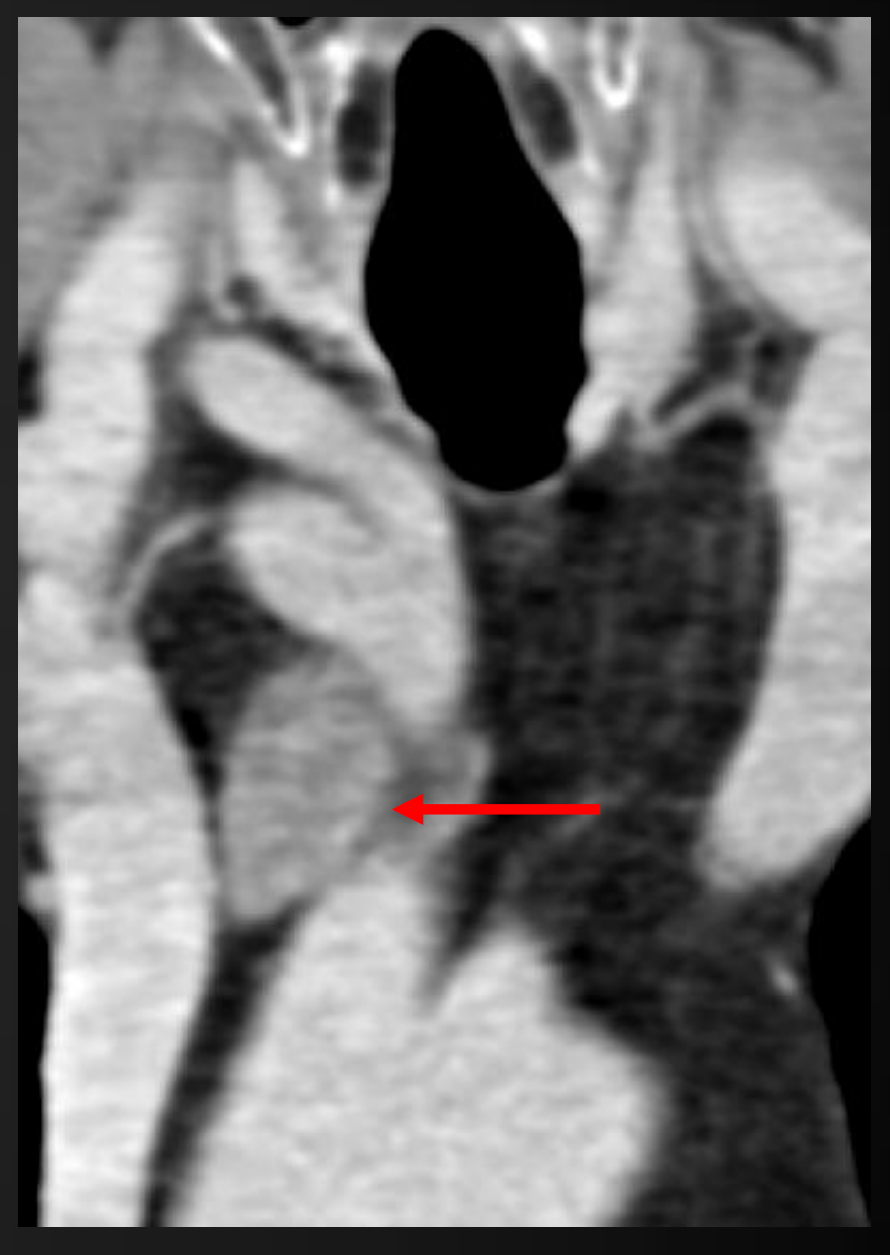

UCLA Radiology
UCLA Health 


\section{Case 5}

- Right sided mediastinal / intrathymic parathyroid adenoma

- Along posterior margin of the right clavicular head

- Hypodense to thyroid on precontrast (A, D), arterial enhancement with cystic change $(B, E)$, and venous washout $(C, F)$

- Can be easy to overlook on axial alone (A-C)

- Best identified on coronal reformats (D-F) 
Case 6

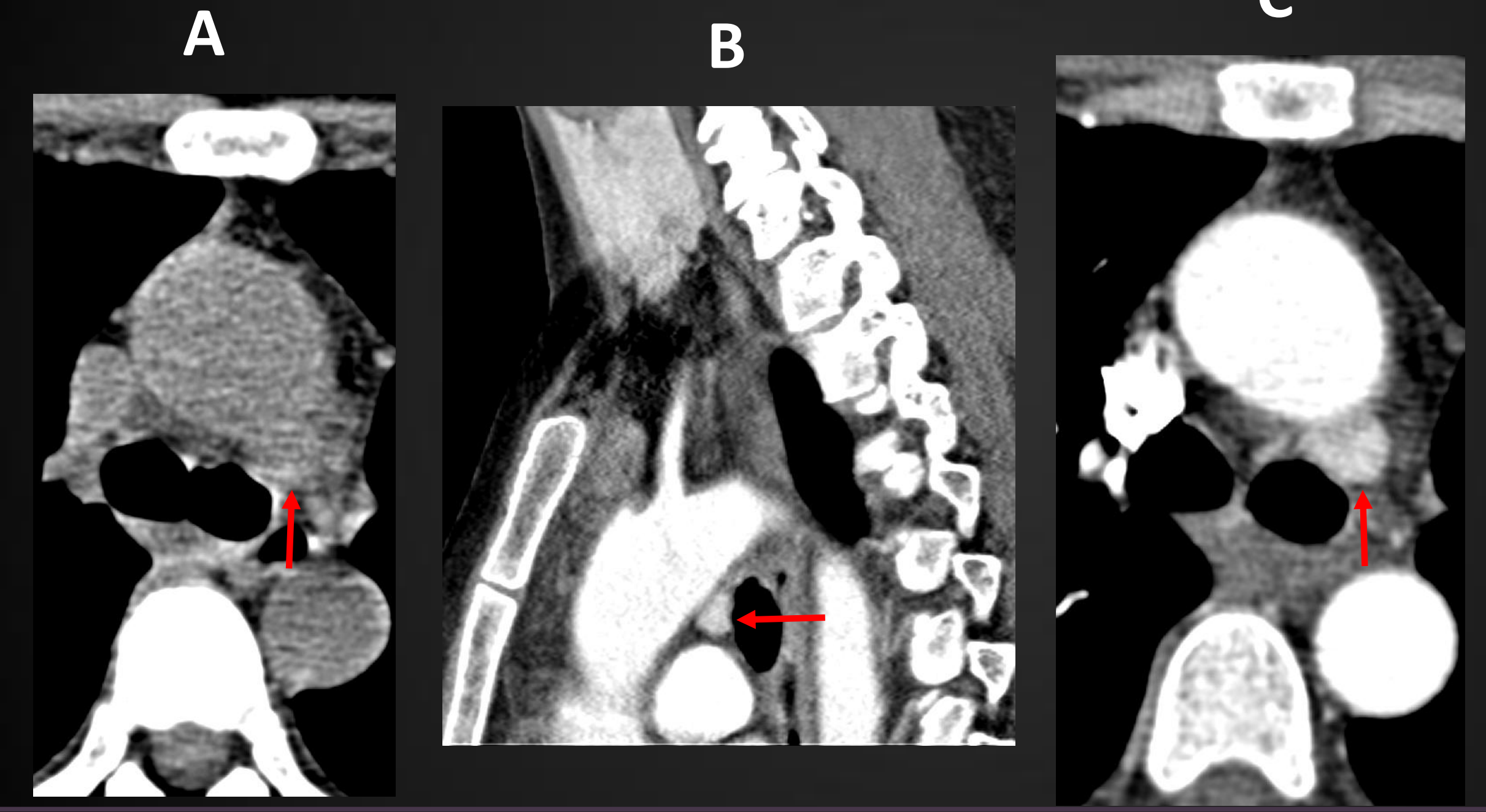

C
D

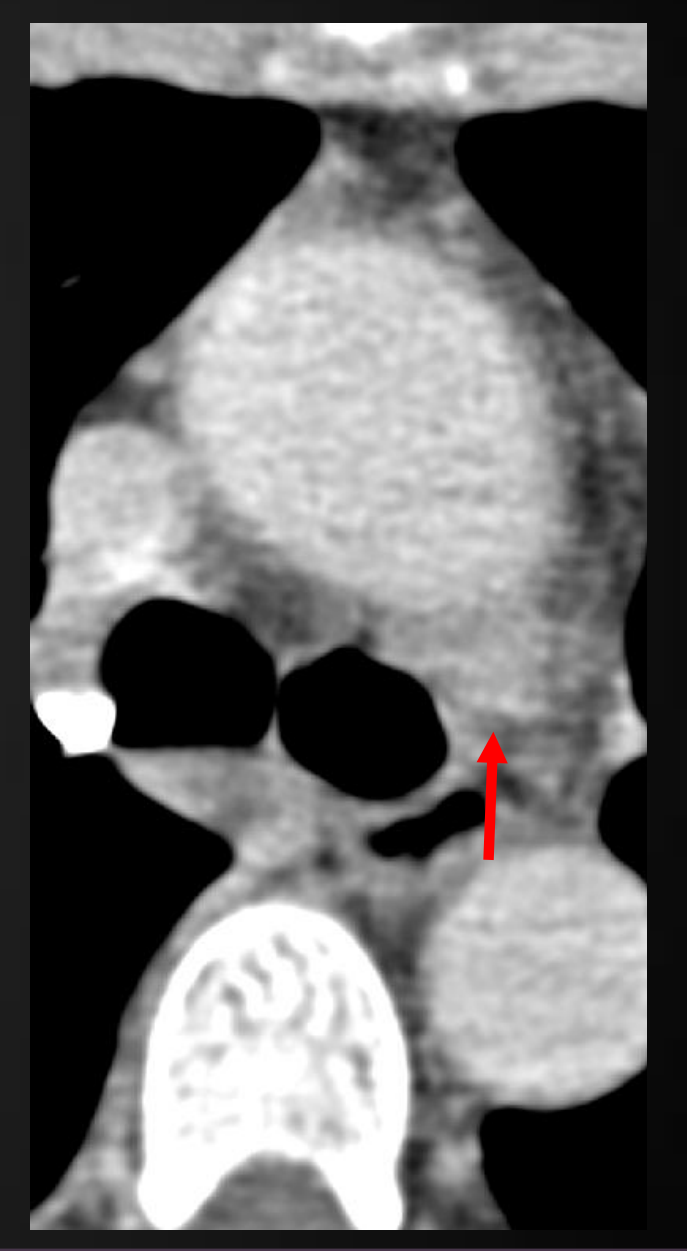

$\underset{\text { UCLA Radiology }}{\text { UCLA Health }}$ 


\section{Case 6}

- Mediastinal (AP window) parathyroid adenoma

- Nodule in the AP window that is hypodense to thyroid on precontrast (A), arterially enhancing (B, C), and demonstrates venous washout (D)

- Remember to look all the way down to the AP window for mediastinal adenomas

- The inferior parathyroid gland migrates with the thymus and can be seen as low as the AP window / carinal level 


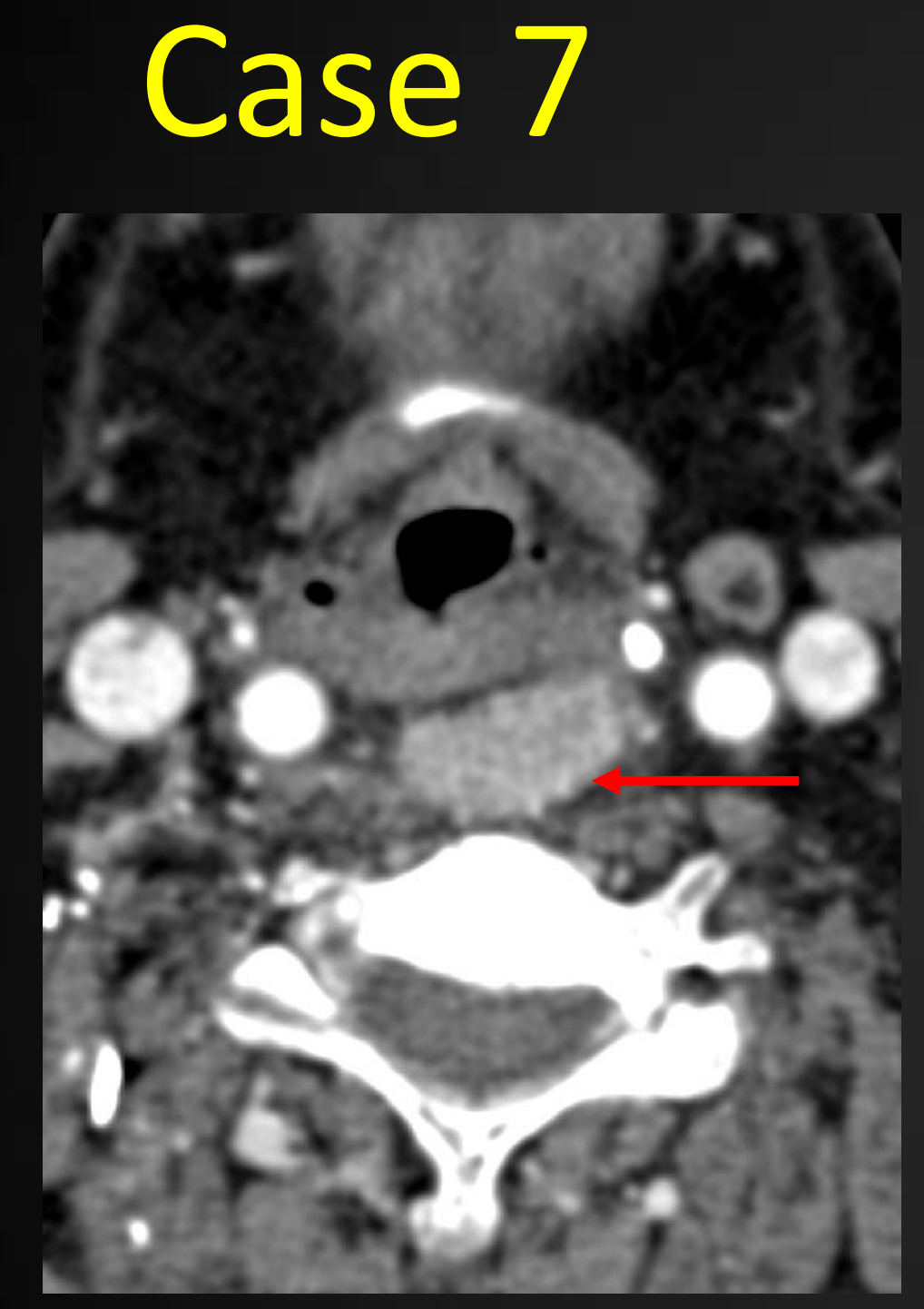
A

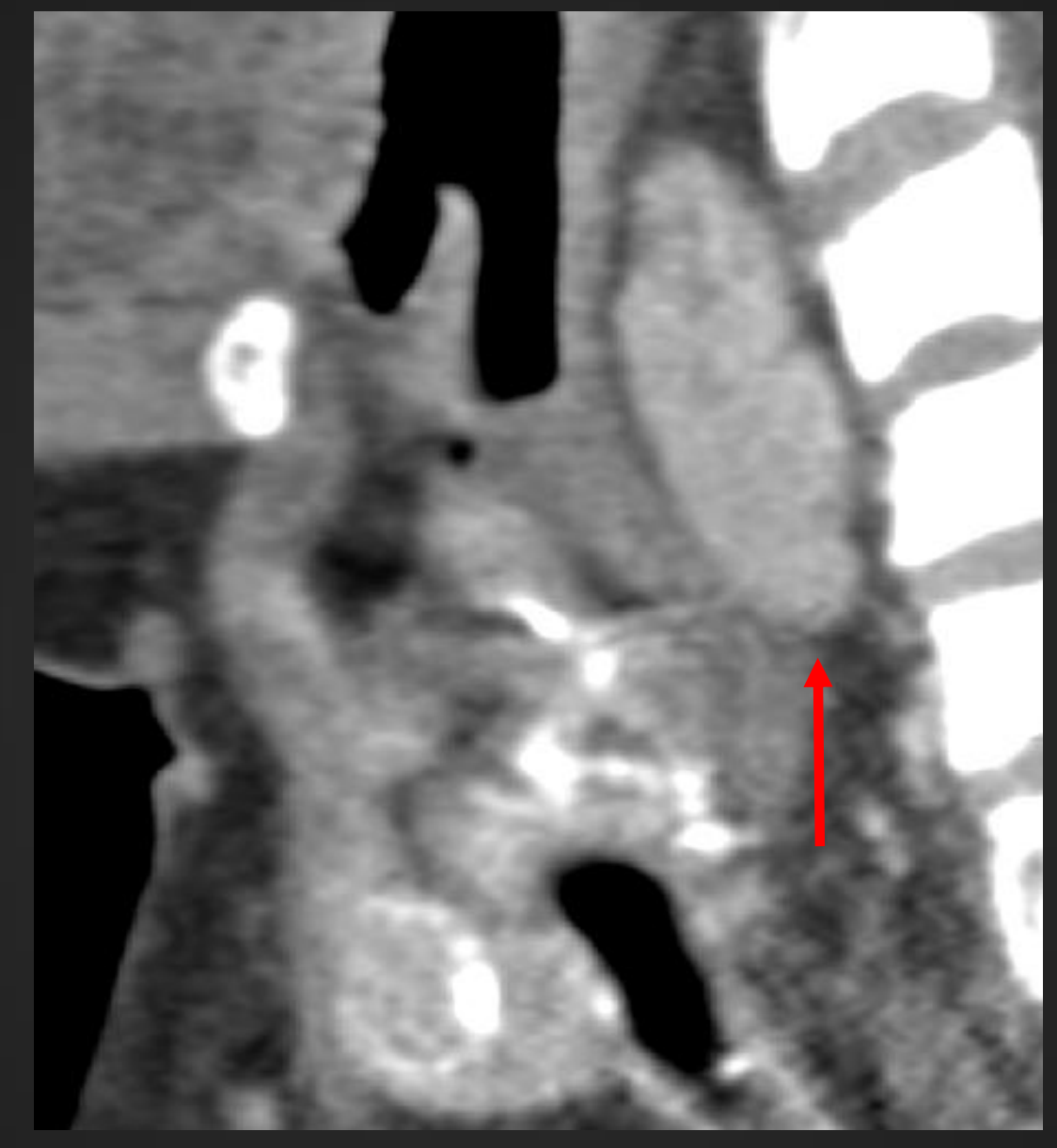

B

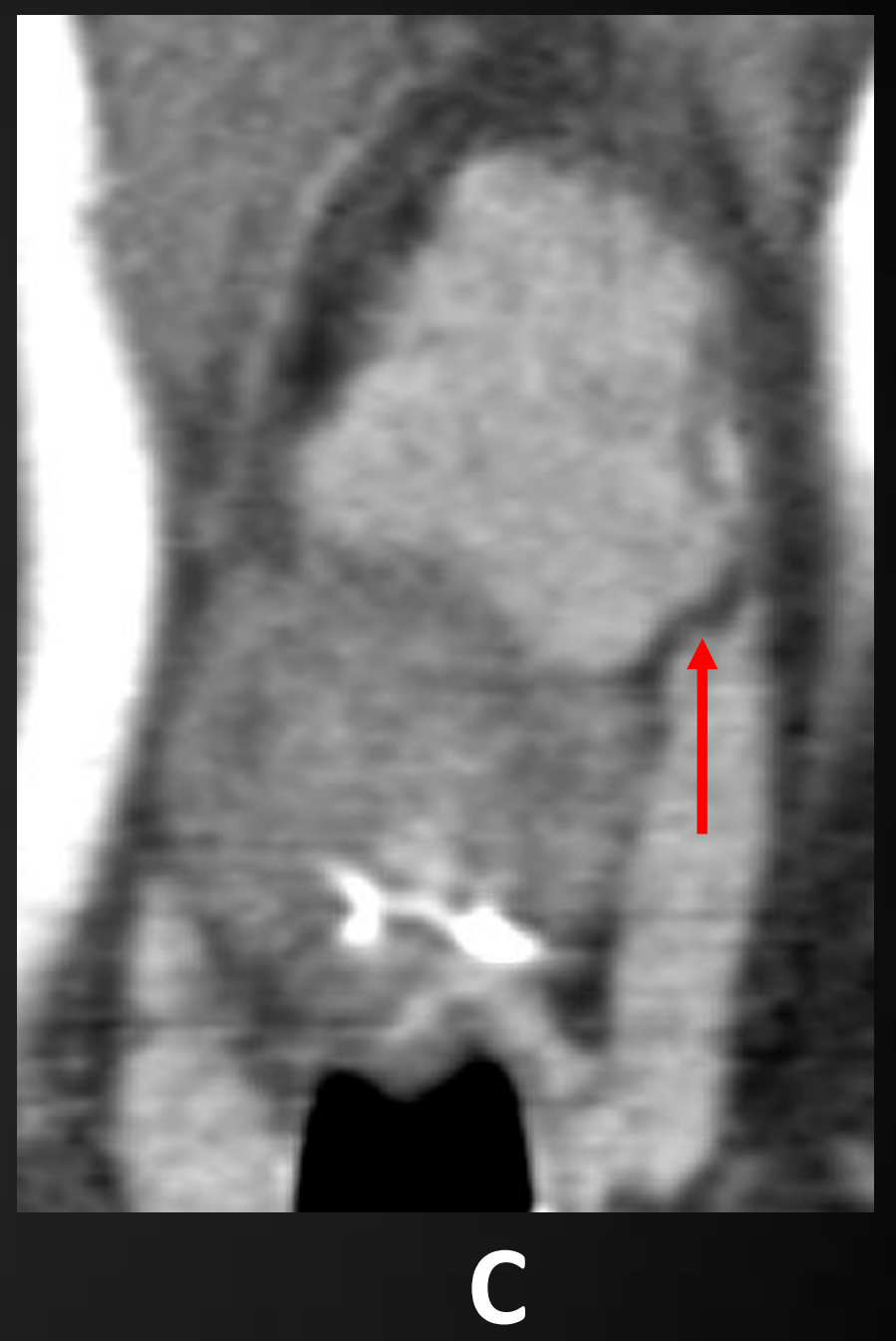

UCLA Radiology 


\section{Case 7}

- Ectopic retropharyngeal parathyroid adenoma at the level of the supraglottic larynx

- Large hyperenhancing mass is seen on axial (A), sagittal (B) and coronal (C) aterial phase images 
Case 8

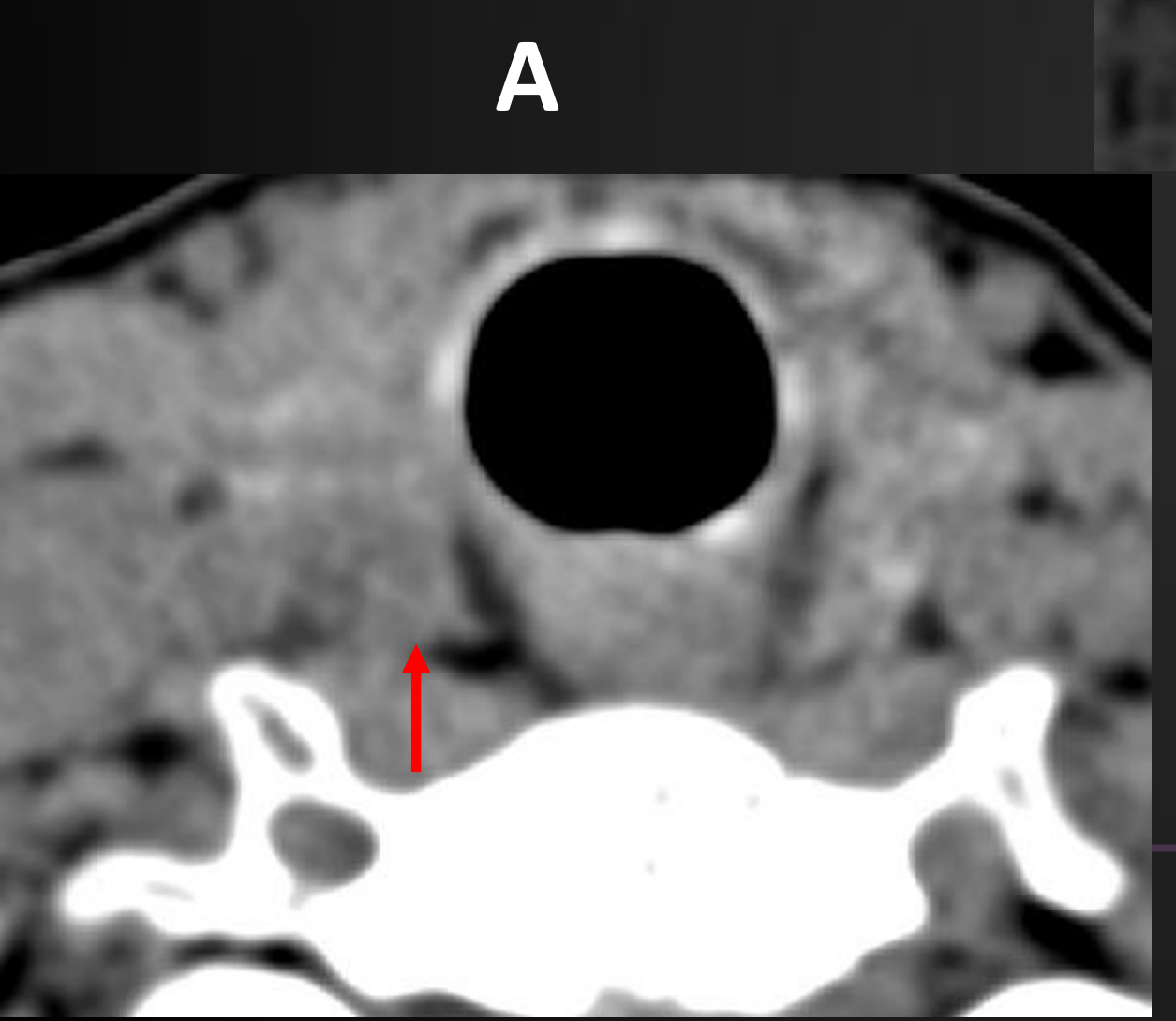

C

B

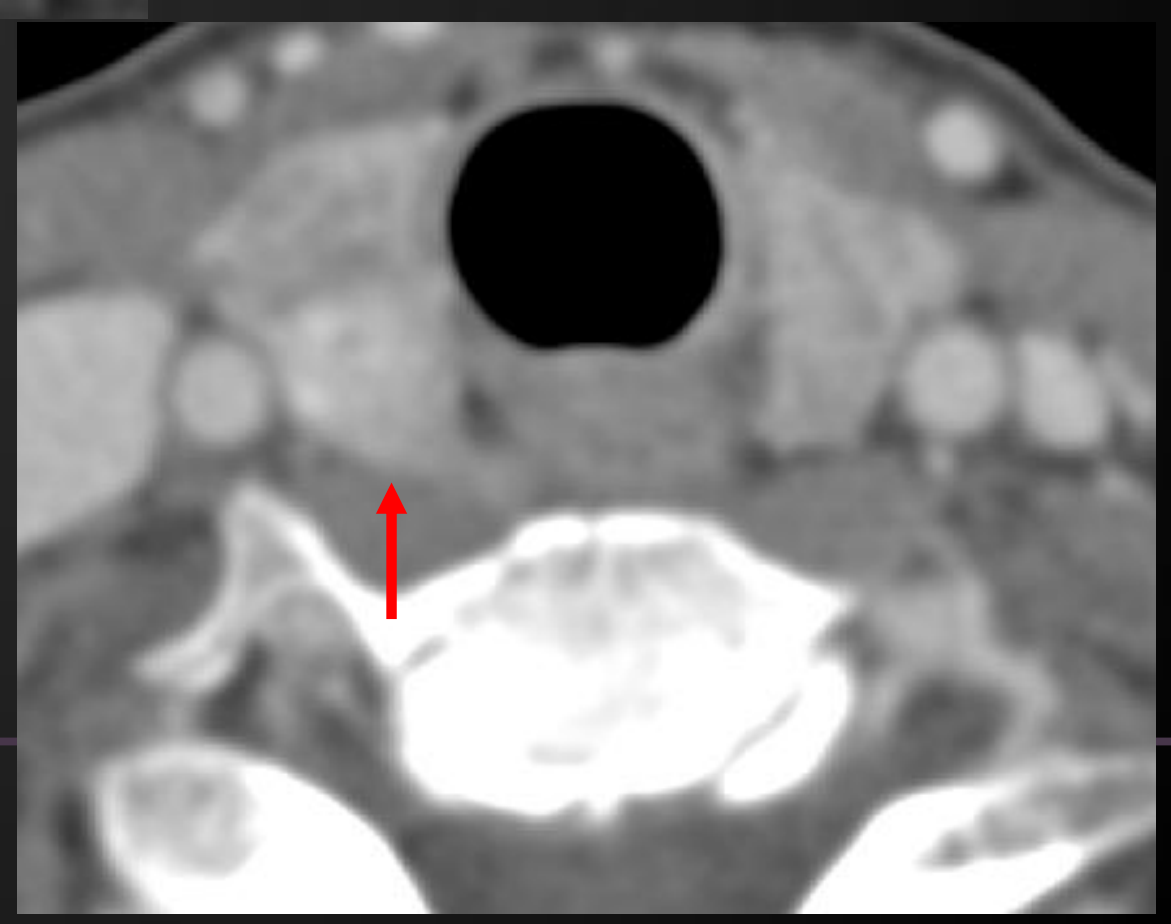




\section{Case 8}

- Right sided intrathyroidal subcapsular parathyroid adenoma

- Nodule along the posterior subcapsular aspect of the right thyroid lobe is hypodense on precontrast $(A)$, hyperenhancing on arterial phase(B), and washes out on delayed phase (C)

- Note background heterogeneity of the thyroid gland 


\section{Case 9}

B

C

D
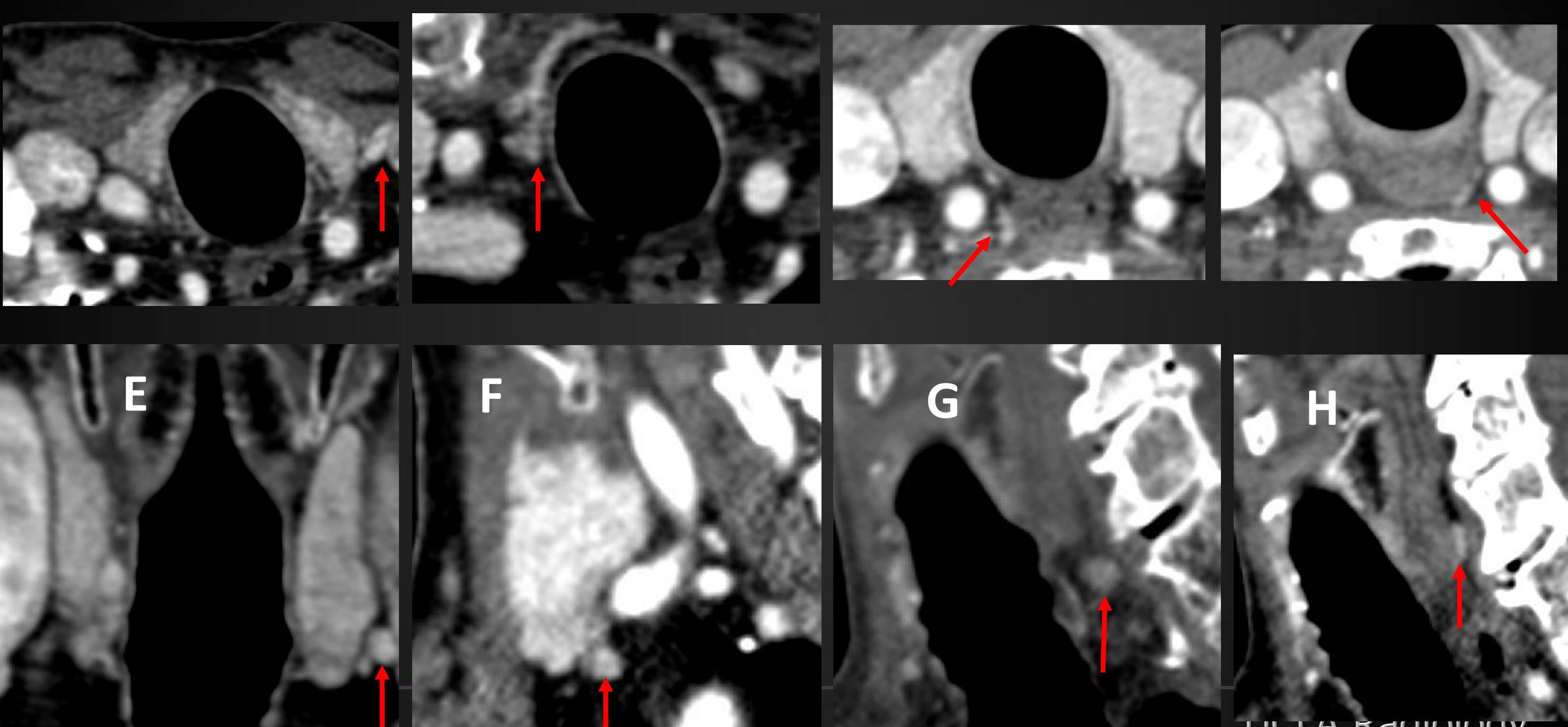

E
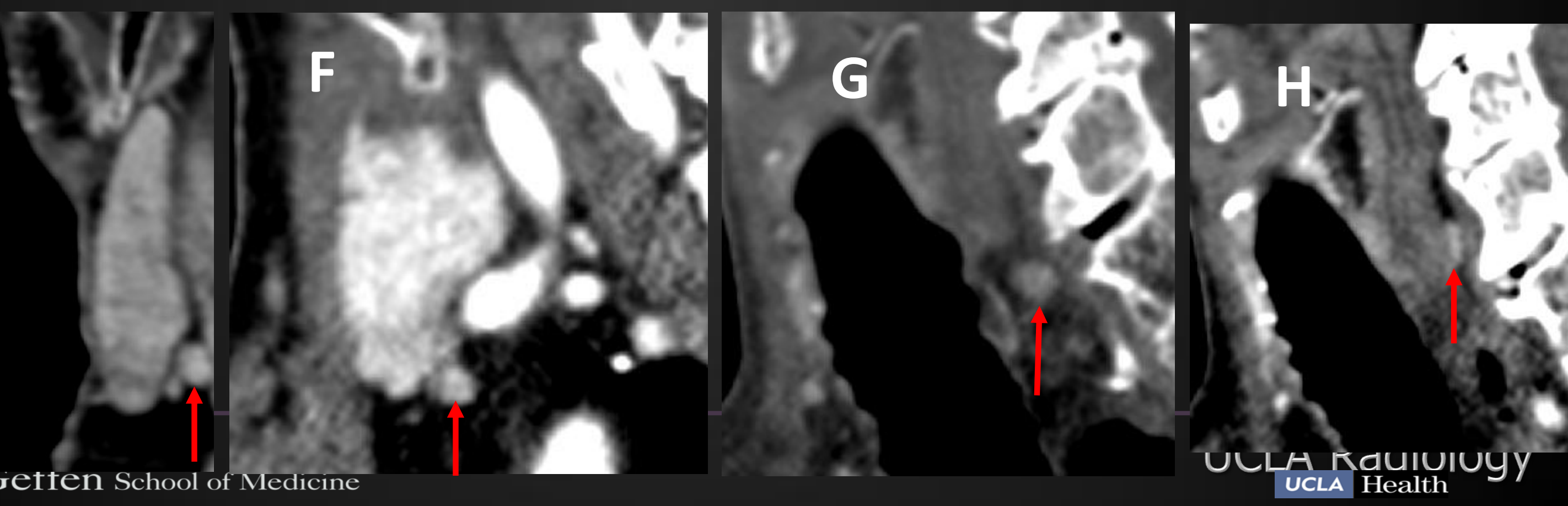


\section{Case 9}

- Multigland hyperplasia

- All four embryologic glands are mildly enlarged:

- Left inferior gland hyperplasia on axial $(A)$ and coronal $(E)$ images

- Deep to the strap muscles, along inferior aspect of the left thyroid lobe

- Right inferior gland hyperplasia on axial (B) and sagittal (F) images

- Paratracheal, along inferior aspect of right thyroid lobe

- Right superior gland hyperplasia on axial (C) and sagittal (G) images

- Paraesophageal, along superior aspect of right thyroid lobe

- Left superior gland hyperplasia on axial (D) and sagittal (H) images

- Paraesophageal, along superior aspect of the left thyroid lobe 


\section{Case 10 - Which image shows the adenoma?}

Axial arterial phase

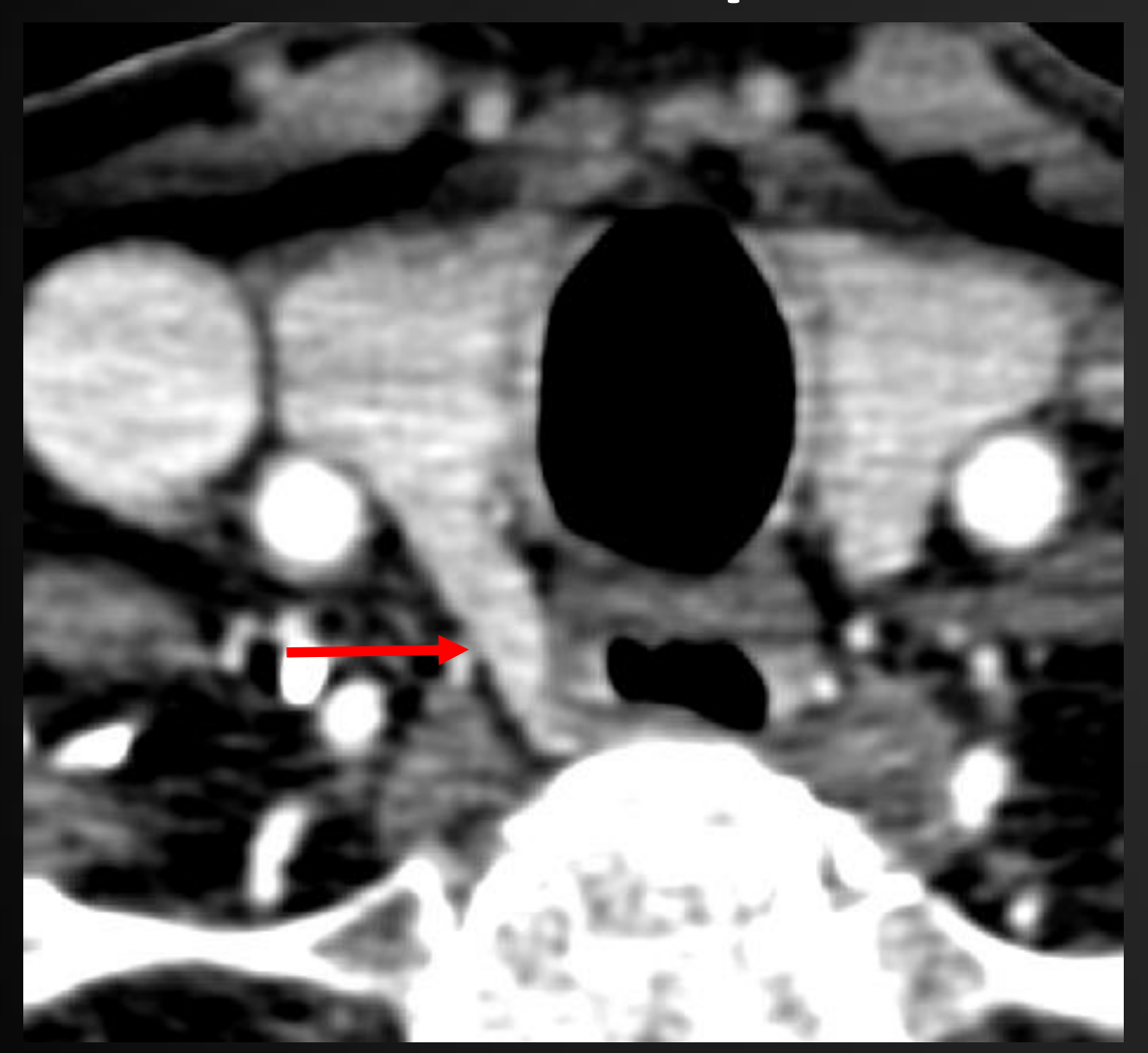

Axial arterial phase

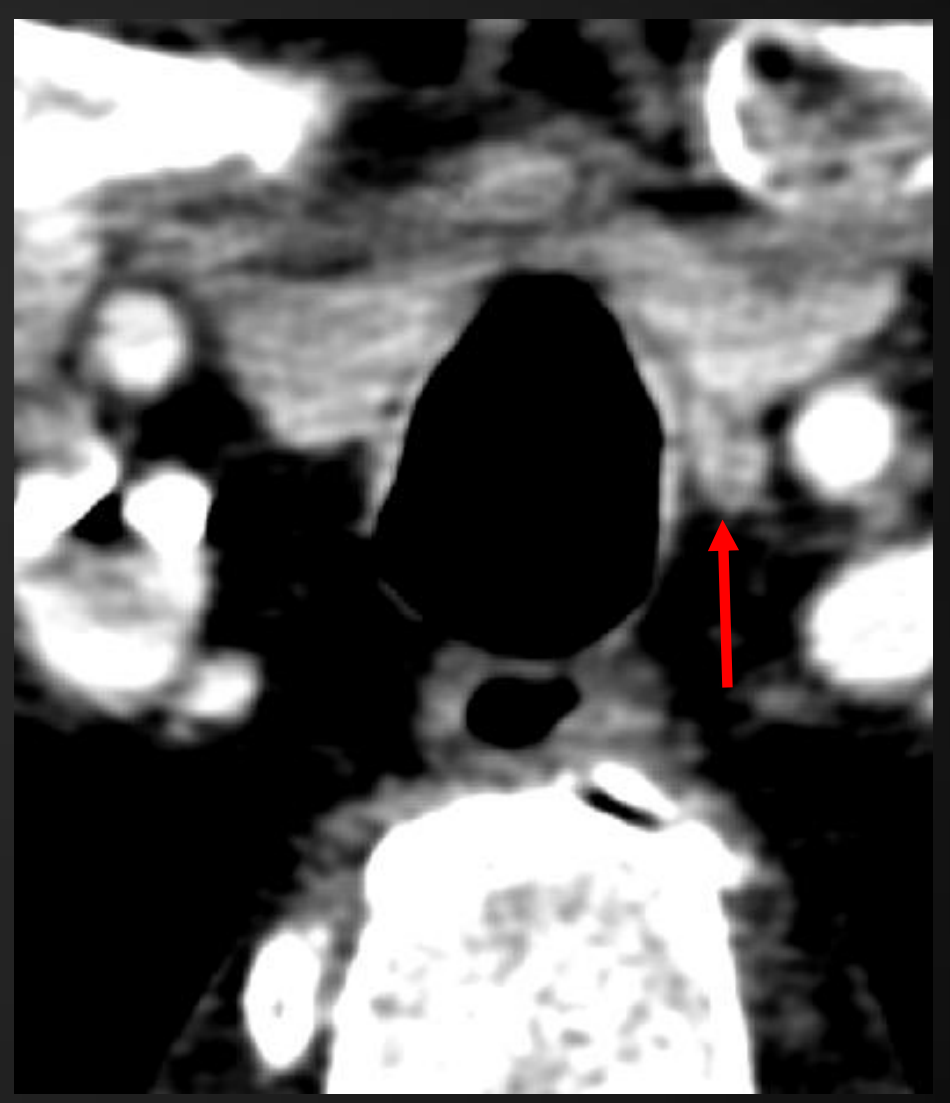




\section{Case 10 - Importance of Precontrast}

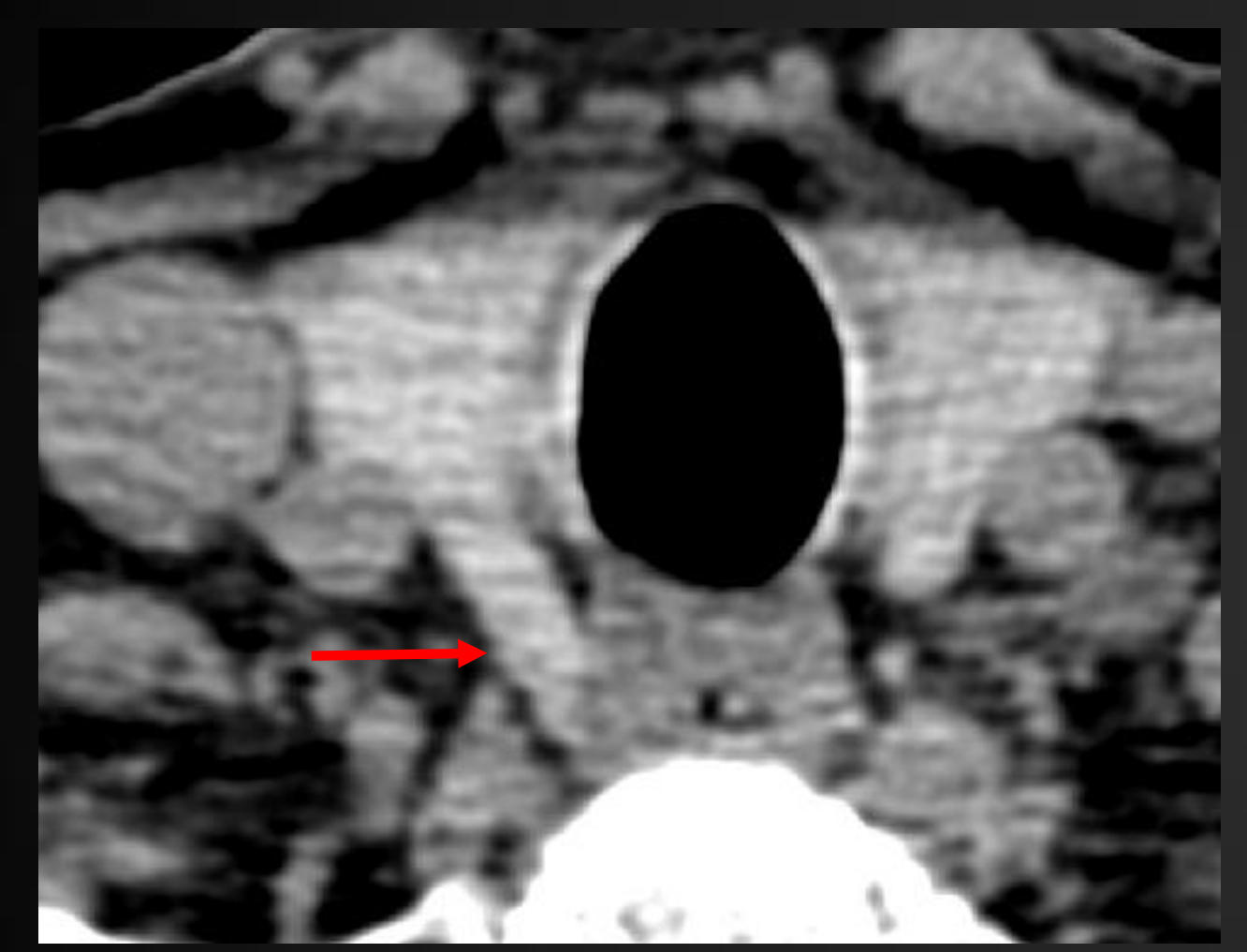

Axial precontrast: Right TE groove nodule isodense to thyroid, thus exophytic thyroid tissue (Zuckercandl tubercle of thyroid)

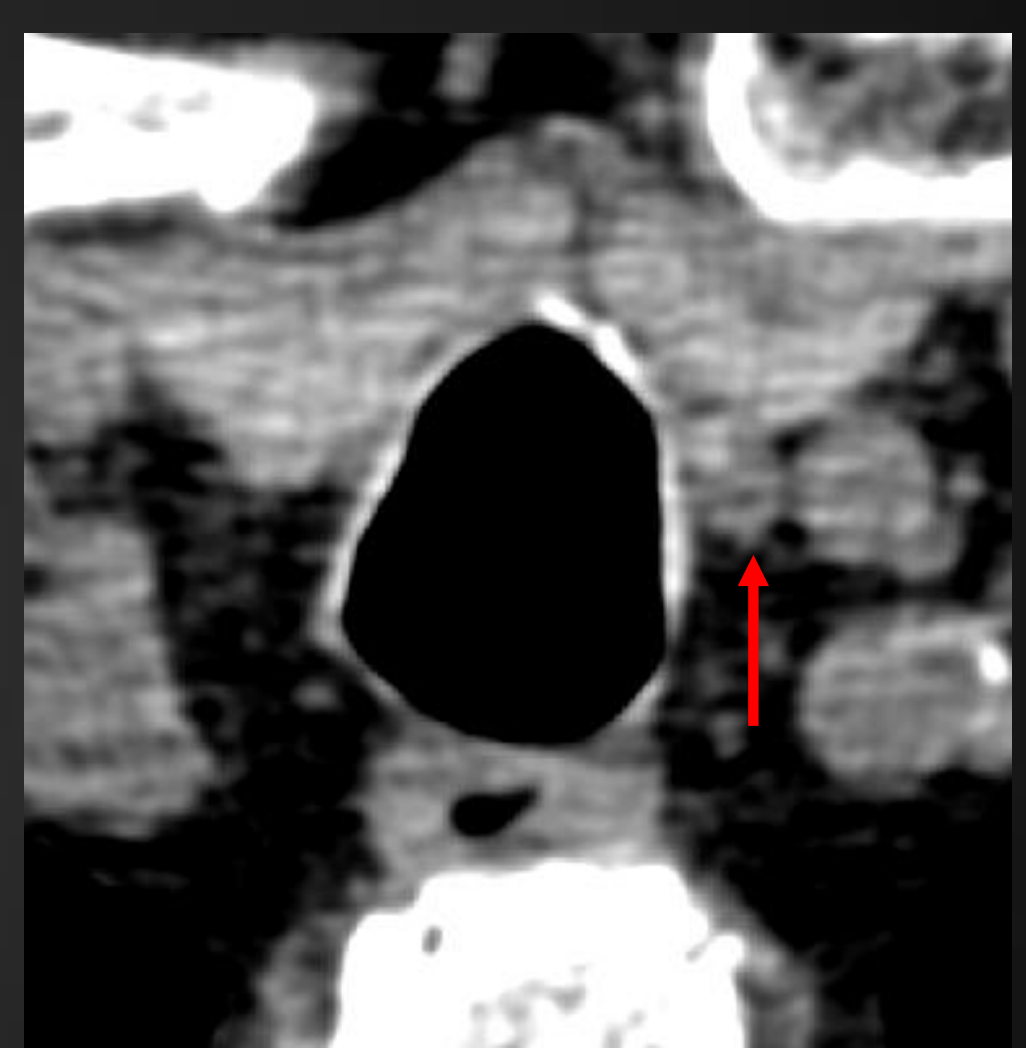

Axial precontrast: Left paratracheal nodule hypodense to thyroid on precontrast, therefore parathyroid adenoma 


\section{Case 10}

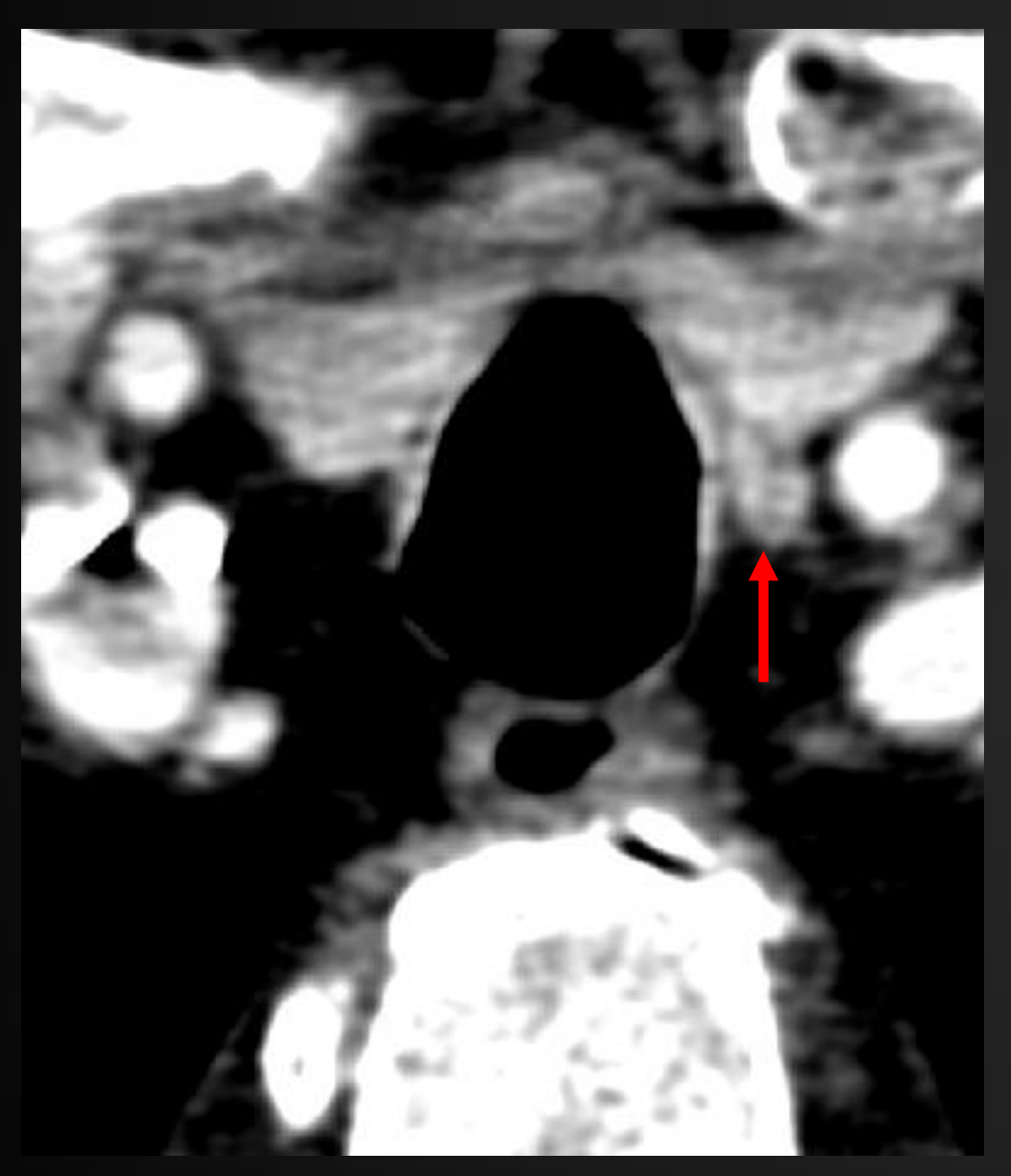

-Left sided embryologic inferior parathyroid adenoma

-Seen along the inferior margin of the thyroid lobe

-Often paratracheal or closely associated with the strap musculature 


\section{Case 10}
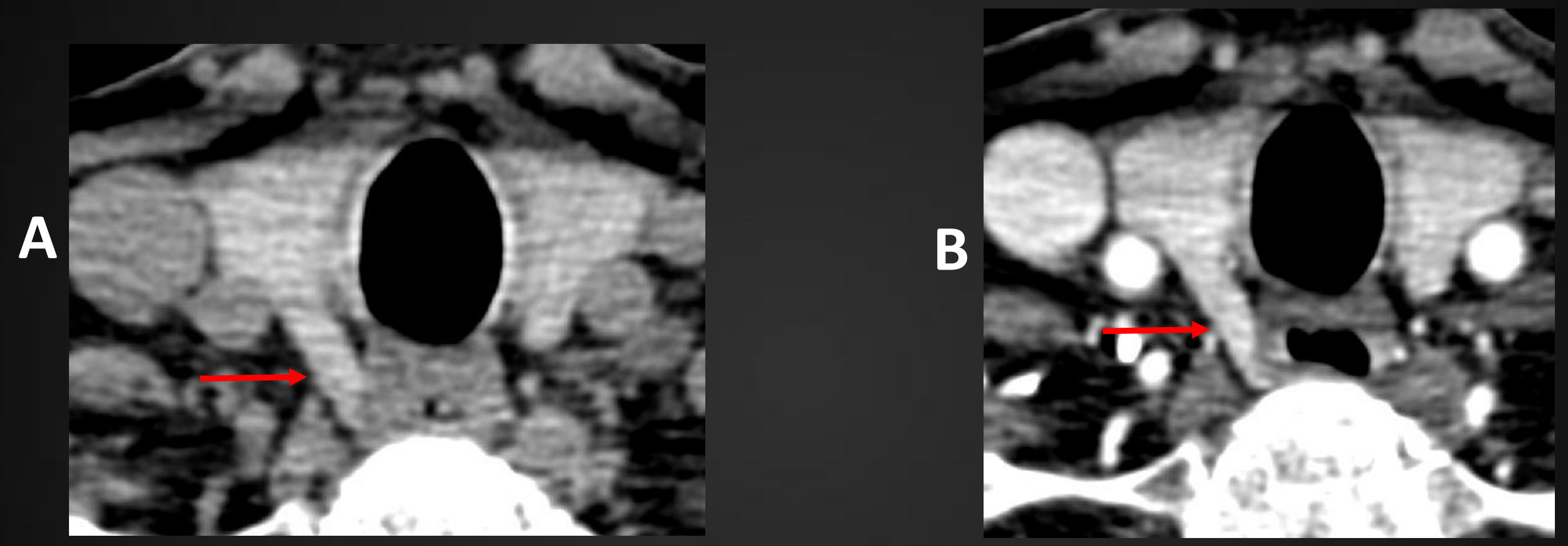

Zuckercandl tubercle. Right sided exophytic thyroid tissue in the tracheoesophageal groove, an anatomic variant characterized by tissue that is isodense to thyroid on precontrast phase $(\mathrm{A})$ and iso-enhancing to thyroid on arterial phase (B) 
Case 11

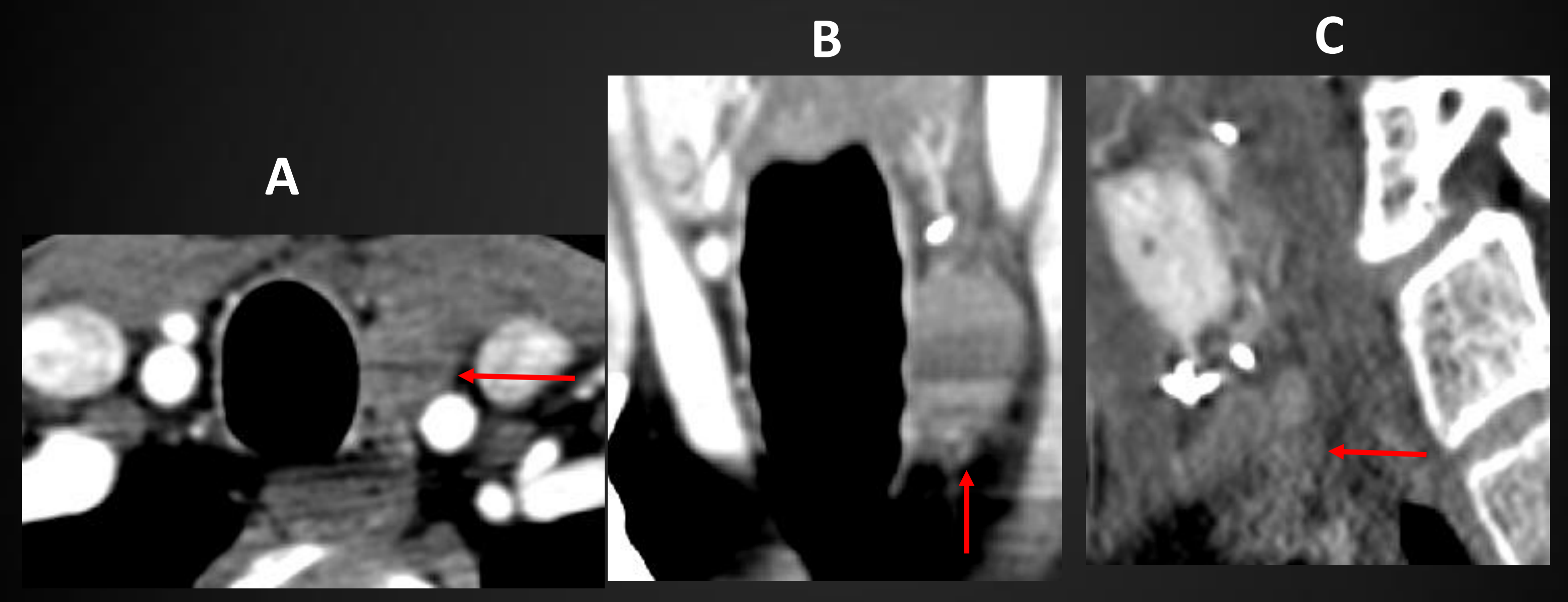




\section{Case 11}

- Parathyroid carcinoma

- Recurrent primary hyperparathyroidism after parathyroidectomy

- Poorly defined hypoenhancing mass with irregular borders in the parathyroidectomy bed on arterial phase axial (A), coronal (B) and sagittal (C) images

- Surgical clips from prior parathyroidectomy 


\section{Case 12}

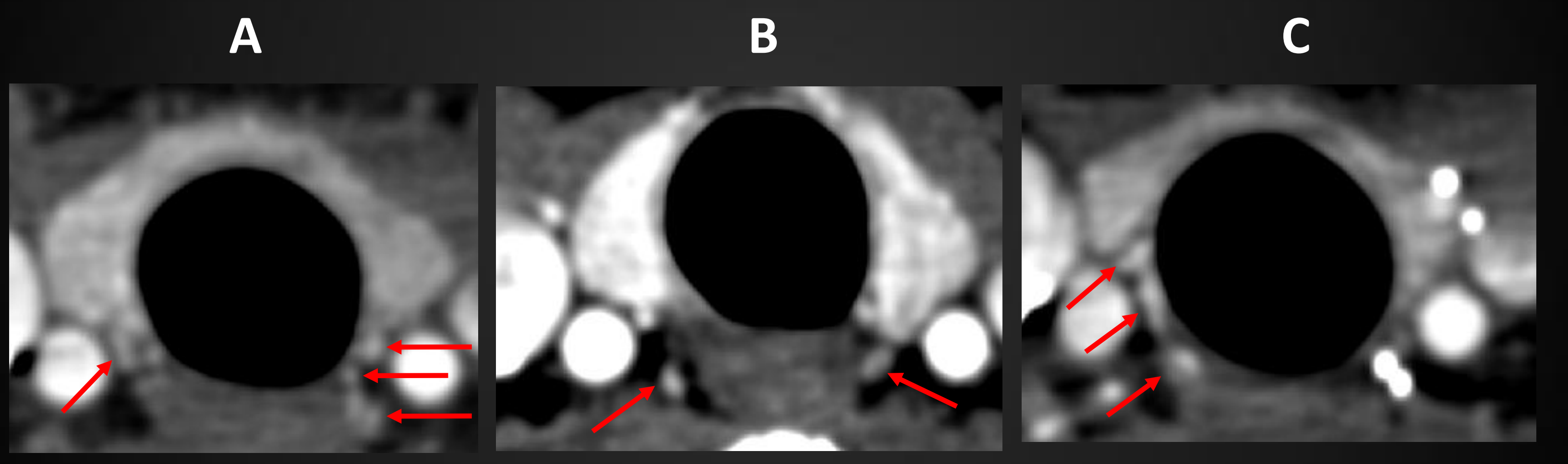




\section{Case 12}

- Parathyromatosis after parathyroidectomy

- Multiple tiny nodules bilaterally in the tracheo-esophageal grooves seen on axial arterial phase images (A-C) .

- Surgical clips from prior parathyroidectomy 
Case 13

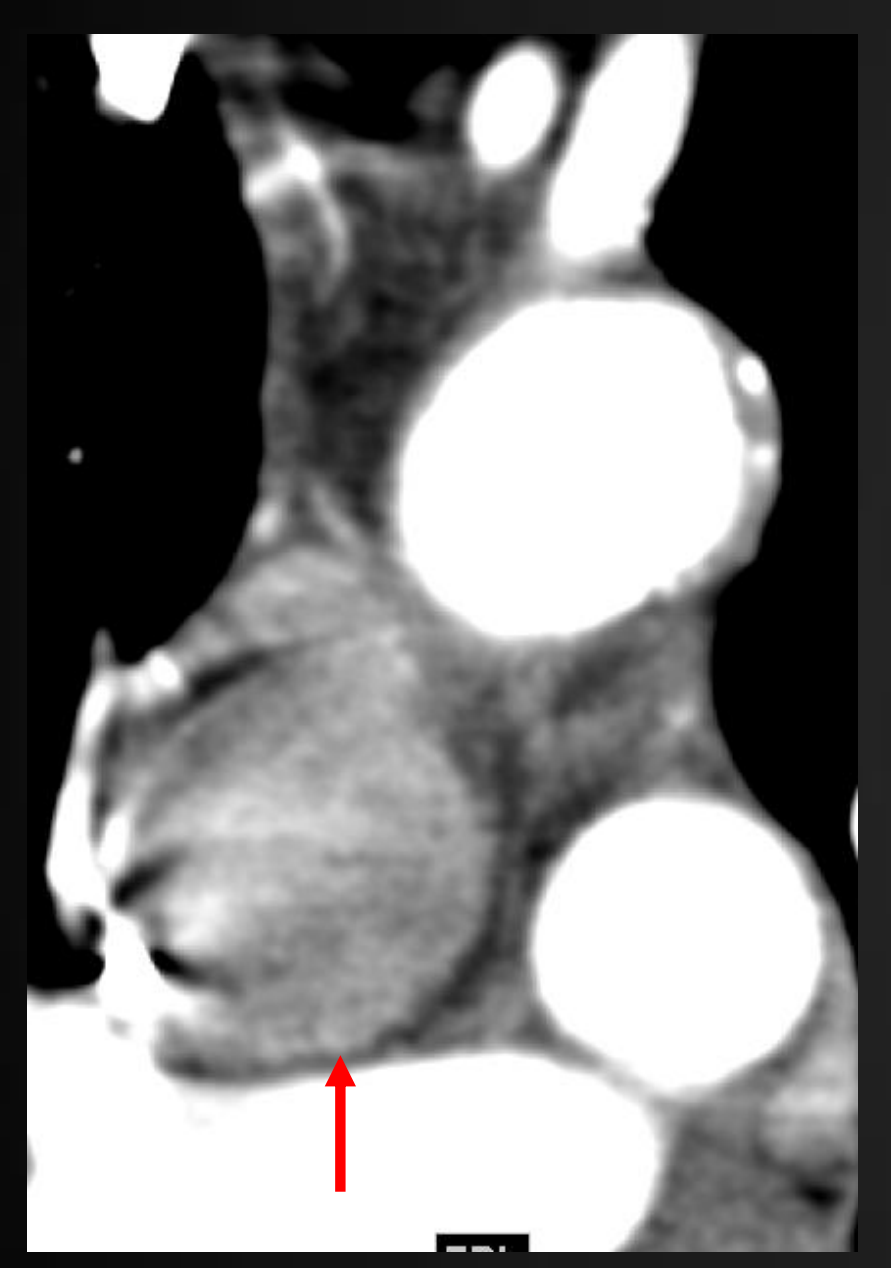

A

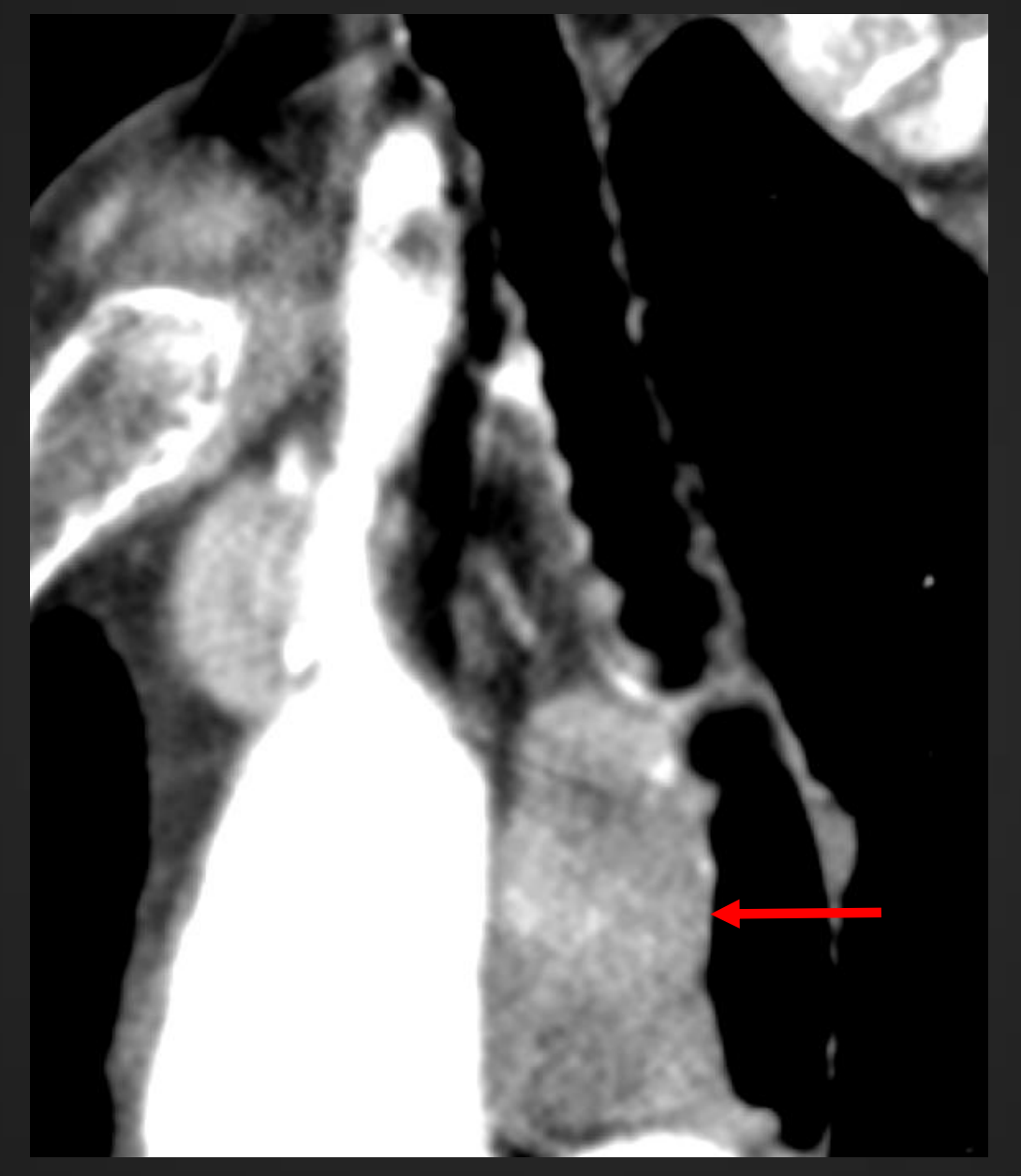

B

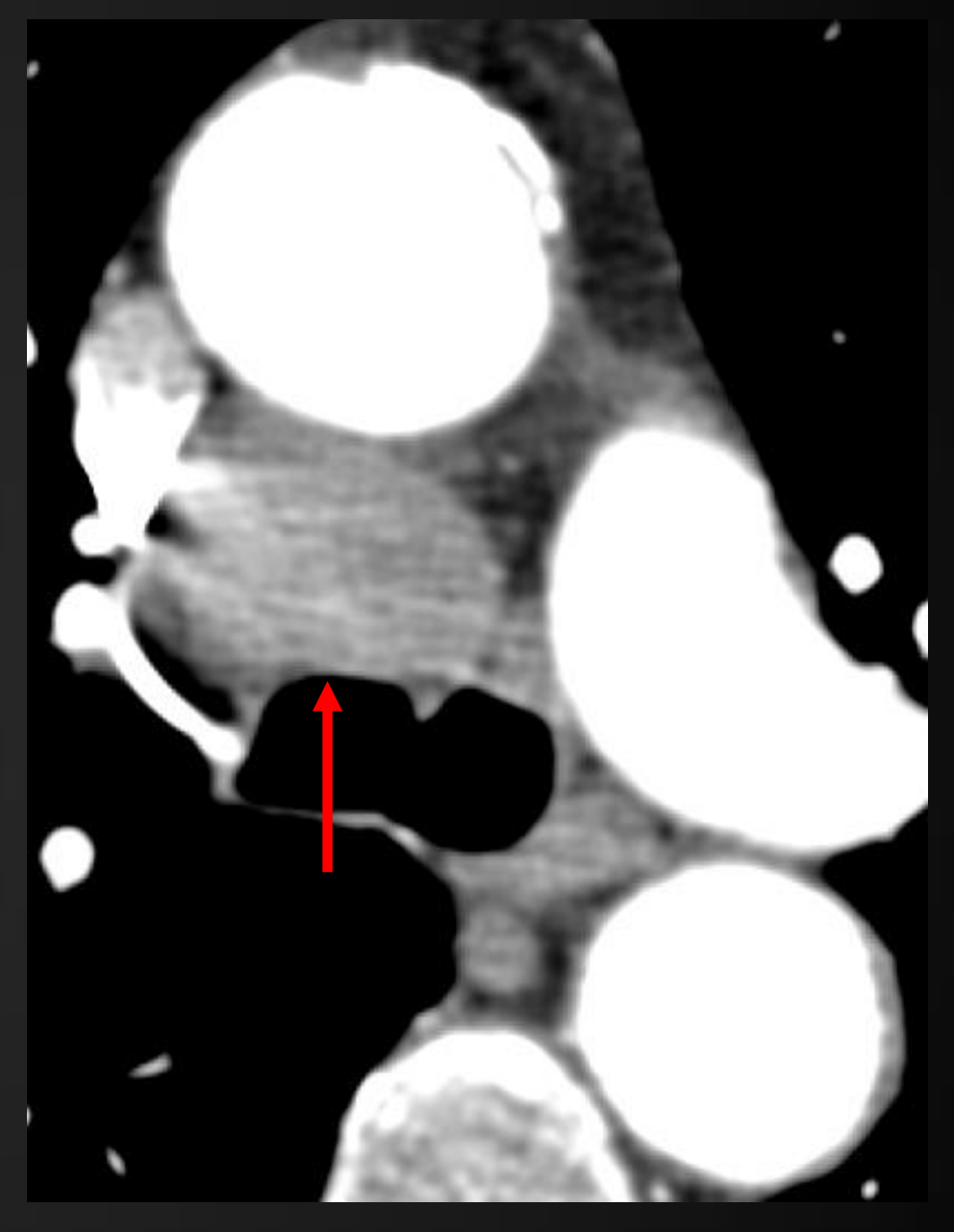

C 


\section{Case 13}

- Biopsy confirmed poorly differentiated carcinoma of unknown primary

- Precarinal mass with only mild enhancement on arterial phase coronal (A), sagittal (B), and axial (C) images 
Case 14 Sestamibi immediate

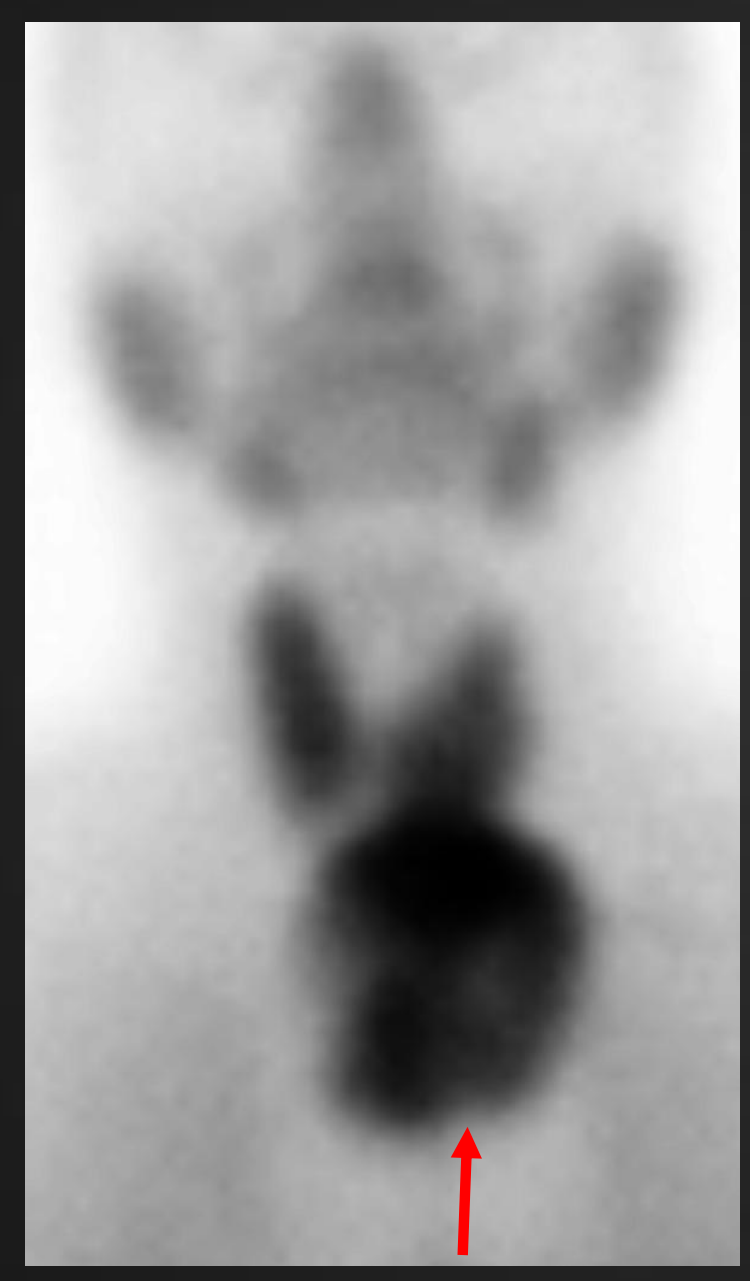

Sestamibi delay 
Case 14

A

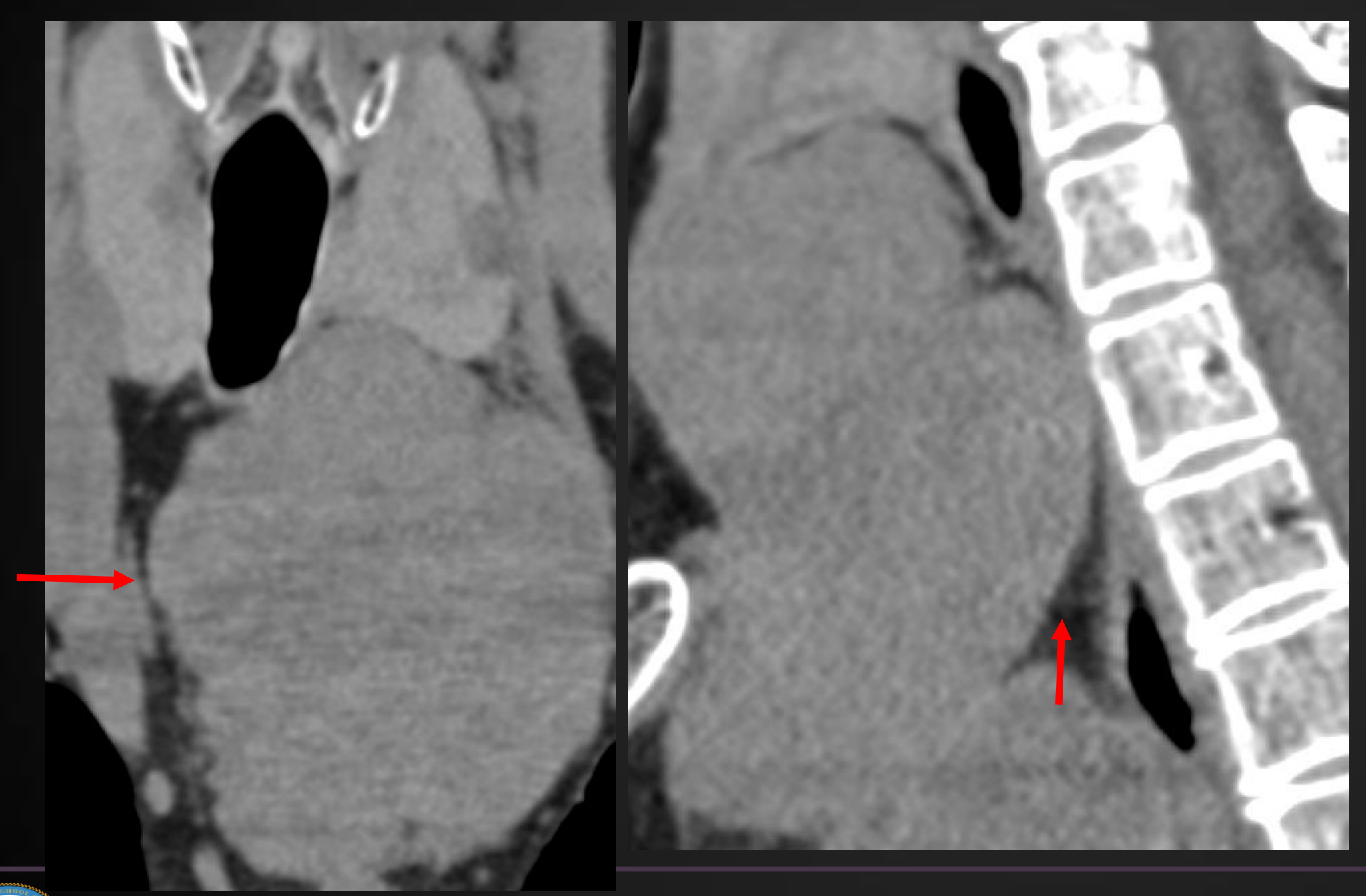

C

\begin{abstract}
B
\end{abstract}

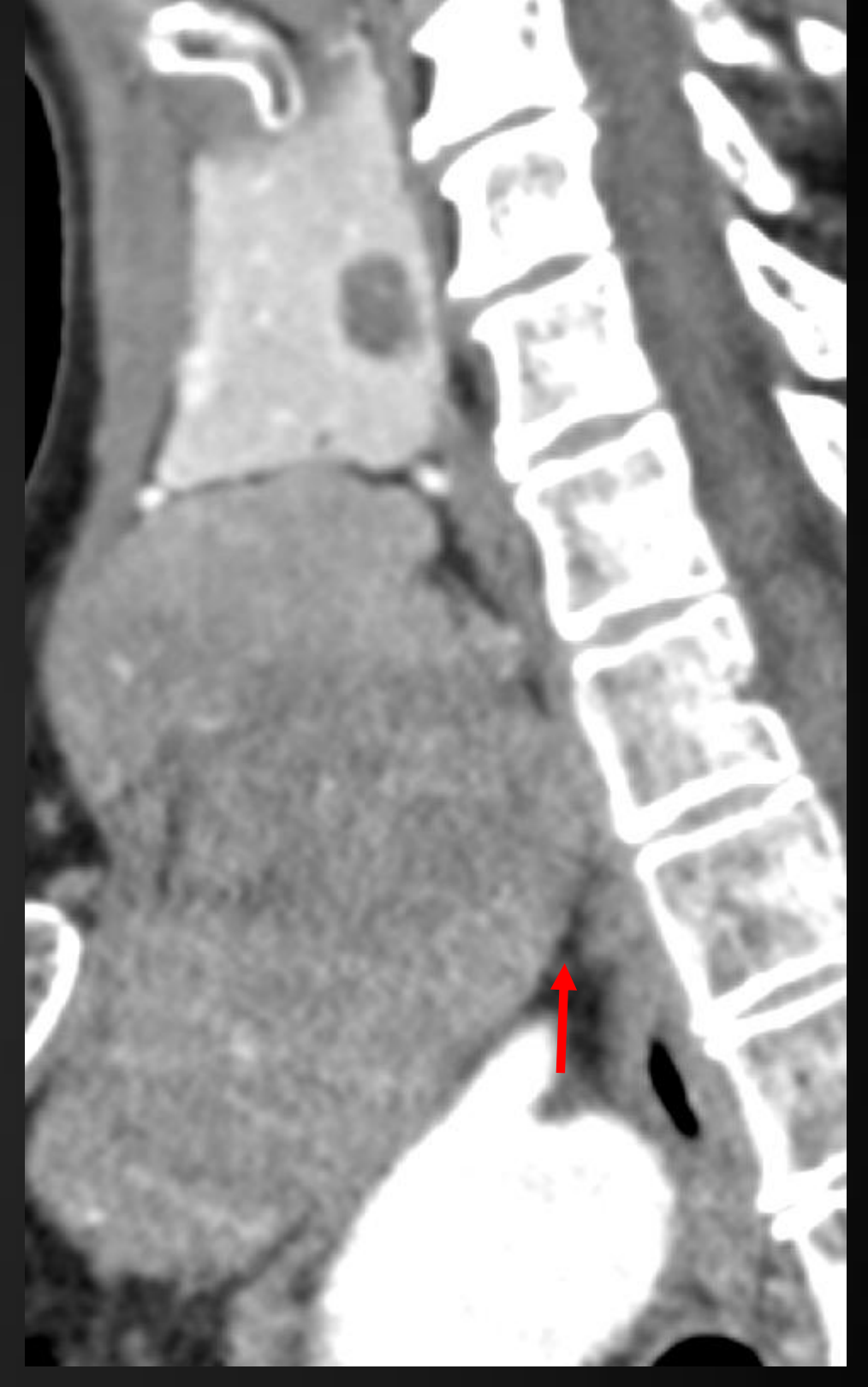

$\underset{\text { UCLA Radioliology }}{\text { UCh }}$ 


\section{Case 14}

\section{- Thymoma}

- Large lobulated anterior mediastinal mass

- Can mimic parathyroid adenoma on Sestamibi parathyroid or myocardial perfusion scans

- Demonstrates increased uptake on the immediate phase Sestamibi with persistent uptake on delayed phase (same as parathyroid adenoma)

- Hypodense to thyroid on precontrast coronal and sagittal images (A, B) and hypoenhancing on arterial phase sagittal (C) images

- Can have areas of calcification and cystic/necrotic change 


\section{Case 15}
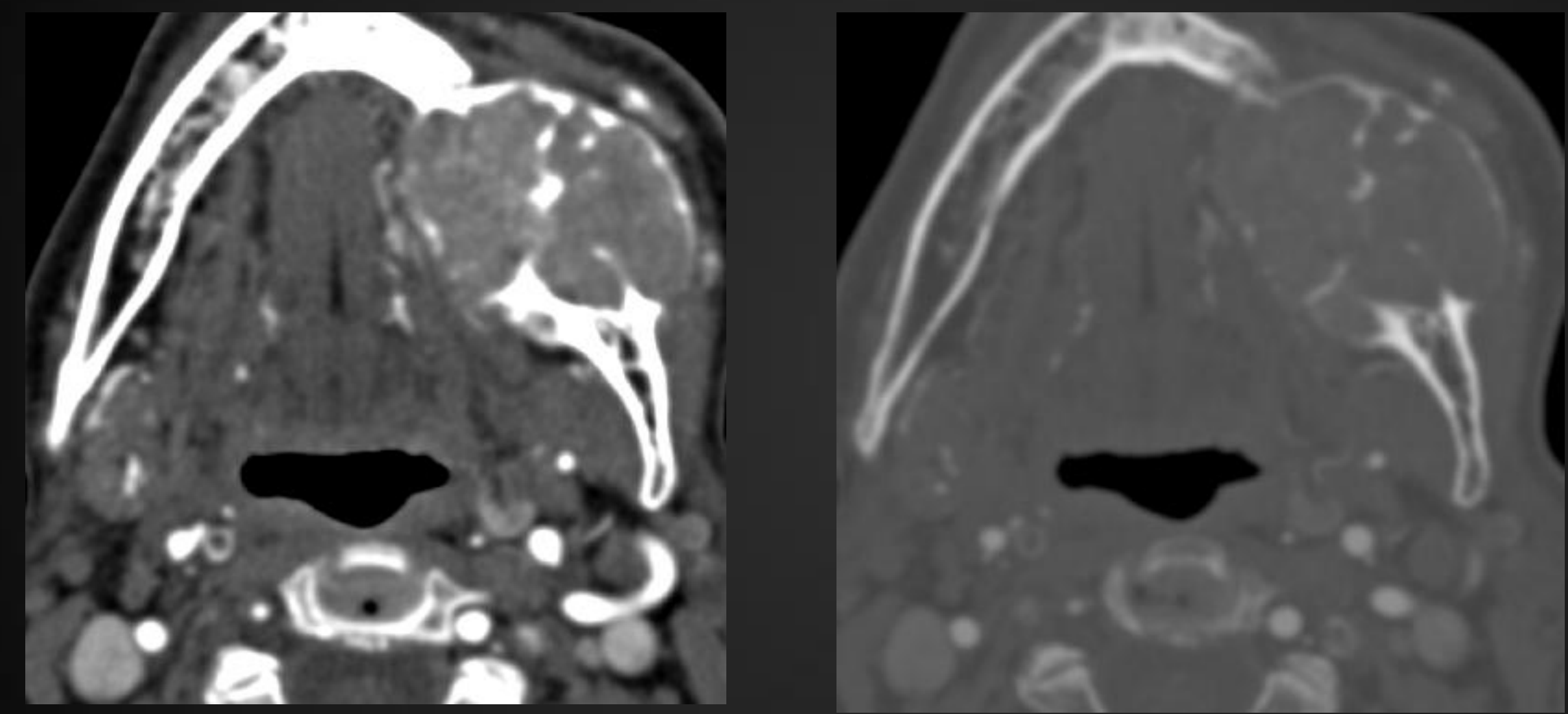


\section{Case 15}

- Brown tumor of the mandible

- Expansile, lytic mass with internal calcification and internal hypderdense components (representing hemorrhage) in the left mandibular body related to primary hyperparathyroidism 


\section{Case 16}
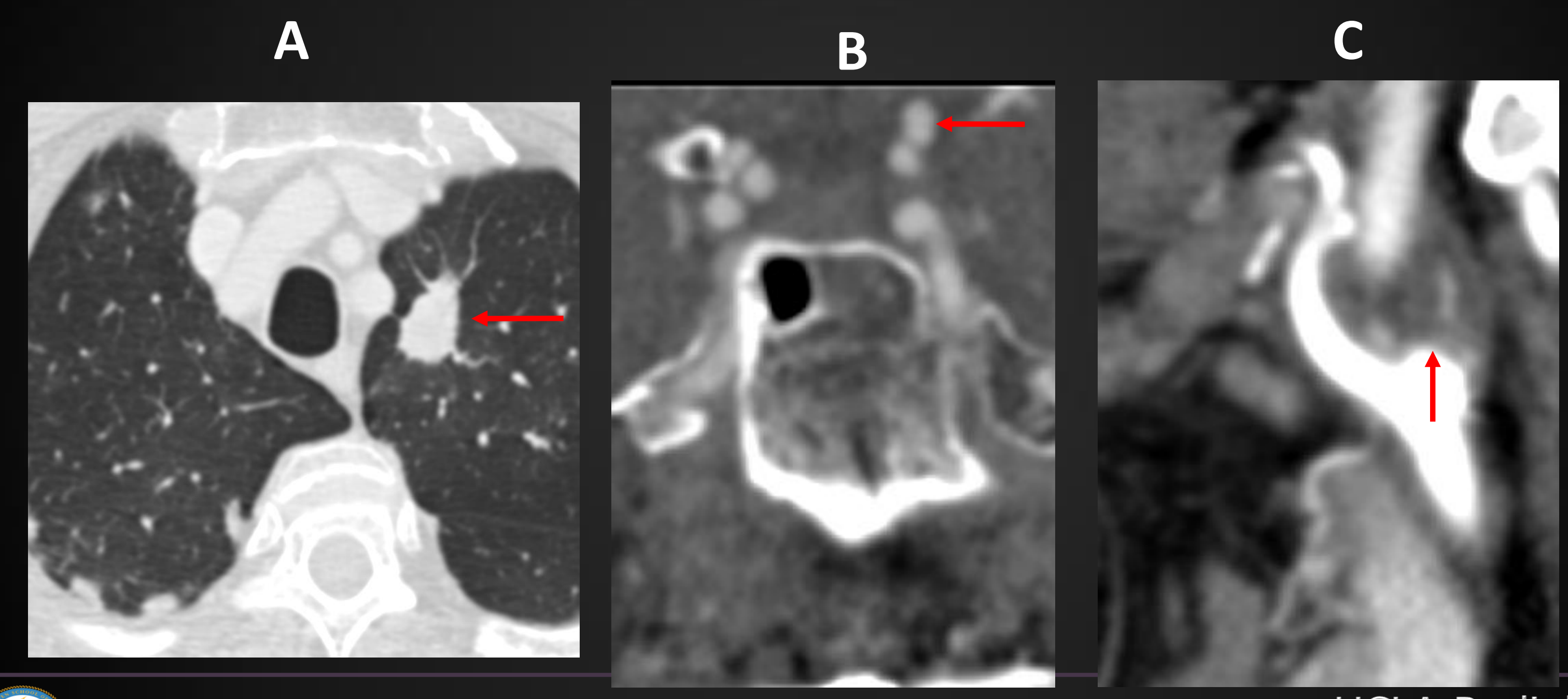


\section{Case 16}

- Commonly encountered incidental findings

- Spiculated left upper lobe mass compatible with pulmonary neoplasm on axial lung window $\mathrm{CT}$ (A)

- Superiorly directed left supraclinoid internal carotid artery aneurysm on axial arterial phase (B)

- Proximal internal carotid artery occlusion on sagittal arterial phase (C) 


\section{Take Home Points}

- Primary Hyperparathyroidism is a relatively common disease associated with significant morbidity.

- Parathyroidectomy is a highly effective and definitive treatment for primary hyperparathyroidisim.

- Accurate preoperative localization is essential in planning for minimally invasive parathyroidectomies.

- 4D CT Parathyroid is emerging as the first line tool for preoperative localization, both for re-operation and initial presentation.

- High quality interpretation of 4D CT examinations should be part of the general radiologist's skillset. 


\section{References}

- Ruda JM, Hollenbeak CS, Stack BC Jr. A systematic review of the diagnosis and treatment of primary hyperparathyroidism from 1995 to 2003. Otolaryngol Head Neck Surg 2005;132(3):359-72.

- Hoang JK, Sung WK, Bahl M, et al. How to perform parathyroid 4D CT: tips and traps for technique and interpretation. Radiology 2014;270(1):15-24.

- Sackett WR, Barraclough B, Reeve TS, et al. Worldwide trends in the surgical treatment of primary hyperparathyroidism in the era of minimally invasive parathyroidectomy. Arch Surg 2002;137(9):105559.

- Rodgers SE, Hunter GJ, Hamberg LM, et al. Improved preoperative planning for directed parathyroidectomy with 4-dimensional computed tomography. Surgery 2006;140(6):932-41.

- Bahl M, Sepahdari AR, Sosa JA, et al. Parathyroid adenomas and hyperplasia on four-dimensional CT scans: three patterns of enhancement relative to the thyroid gland justify a three-phase protocol. Radiology 2015;277(2):454-62.

- Sepahdari AR, Bahl M, Harari A, et al. Predictors of multigland disease in primary hyperparathyroidism: a scoring system with 4D-CT imaging and biochemical markers. Am J Neuroradiol 2015;36: 987-92. 